\title{
Quantum Statistical Model of Nuclear Multifragmentation in the Canonical Ensemble Method
}

\author{
A.S. Parvan $\nmid \S$, V.D. Toneev†‡ and M. Płoszajczak $\ddagger$ \\ $\dagger$ Bogoliubov Laboratory of Theoretical Physics, Joint Institute for Nuclear Research, \\ 141980 Dubna, Russia \\ $\S$ Institute of Applied Physics, Moldova Academy of Sciences, MD-2028 Kishineu, Moldova \\ $\ddagger$ Grand Accélérateur National d'Ions Lourds (GANIL), CEA/DSM - CNRS/IN2P3, BP 5027, \\ F-14076 Caen Cedex 05, France
}

\begin{abstract}
A quantum statistical model of nuclear multifragmentation is proposed. The recurrence equation method used within the canonical ensemble makes the model solvable and transparent to physical assumptions and allows to get results without involving the Monte Carlo technique. The model exhibits the first order phase transition. Quantum statistics effects are clearly seen on the microscopic level of occupation numbers but are almost washed out for global thermodynamic variables and the averaged observables studied. In the latter case, the recurrence relations for multiplicity distributions of both intermediate-mass and all fragments are derived and the specific changes in the shape of multiplicity distributions in the narrow region of the transition temperature is stressed. The temperature domain favorable to search for the HBT effect is noted.
\end{abstract}




\section{INTRODUCTION}

A considerable interest is paid to heavy ion collisions which result in the formation of residual nuclei with the excitation energy about 5-10 $\mathrm{AMeV}$. In this range of excitation energies one can probe an intriguing possibility of the nuclear liquid-gas phase transition. Below these energies the produced system is deexcited by the evaporation of particles, mainly nucleons, as would be expected from a hot liquid drop, whereas at higher excitation energies the excited source explodes with simultaneous formation of many Intermediate Mass Fragments (IMF). The way from the production of a residual nucleus to the formation of a multi-fragment system (the multifragmentation process) is a complex phenomenon where a relative role of dynamical and equilibrium effects is still not completely understood. Such a situation gives rise to the variety of approaches for describing the many-facet nuclear multifragmentation : from an evaporative picture [1] and simple percolation models [2, 3] till a refined statistical treatment [4 [7] and complicated dynamical models [8 12] (see the cited above review-articles and references quoted therein).

In this work we develop the Quantum Statistical Model (QSM) of multifragmentation based on the canonical formalism. Quantum statistical treatment has been applied earlier to the multifragmentation process in Refs. [13 17] but within the grand canonical ensemble providing the conservation of proton and neutron numbers in the mean. The canonical method, which is more suitable for the description of multifragmentation of a finite system, corresponds to the exact conservation of baryon number. However till now it was used only for the case of classical Boltzmann fragments [6, 18, 19]. The mathematical basis of our consideration is the recursive technique [20] which allows one to eliminate the Monte Carlo calculations [6] and to present results in an analytical form being rather similar to that for the Boltzmann case [18,19]. The model developed in this paper addresses the issue of quantum effects in the multifragmentation process. Although these effects a priori cannot be large, in some particular cases they may be important. For example, it was demonstrated that the yield of $L i$-isotopes, which is used in the studies of the caloric curve behavior to determine the system temperature by means of the isotope-ratio method, is sensitive to quantum statistics [17]. Intensity interferometry for identical fragments [21] may give certain information on the size of fragmenting sources what is of primary importance for understanding the multifragmentation process. It is also well-known that Bose-Einstein correlations can be the source of salient short-ranged phase-space correlations [22,23]. In general, the study of quantum systems of identical particles with incomplete information may give intriguing and counterintuitive results even in simple cases as exemplified in [24].

This paper is divided as follows. Thermodynamics of ideal quantum gas of identical particles is considered in Sec. 2. In this section we introduce the method of recursive relations. The canonical ensemble approach is generalized in Sec. 3 to the case of mixture of different quantum gases for a given composition of its components. Based on these results, the QSM is introduced in Sec. 4. Technical details of calculations are given in the Appendixes. The partition function, various thermodynamic quantities as well as multiplicity fragment

distributions and their moments are derived analytically via the recurrence relations. Illustrative numerical results of the QSM are also presented and discussed in this section. Finally, concluding remarks are given in Sec. 5. 


\section{PERFECT GAS OF IDENTICAL PARTICLES}

Let us consider an ideal gas of $N$ identical particles, either bosons or fermions. We want to find the canonical ensemble partition function and the ensemble averaging of particle multiplicity. Our consideration will be based on constructing the recurrence relations which enable an exact calculation of both the statistical partition function and the ensemble averaging [20].

Let a system of ideal bosons (fermions) be enclosed in the volume $V$ at the temperature $T$. Without any loss of generality we can limit ourselves to the case of spinless particles, i.e., bosons and fermions differ only by the symmetry of the wave function with respect to permutation of identical particles. A microscopic state of the system is determined by a set of occupation numbers of single-particle levels $\left\{\nu_{\vec{p}}\right\}$, so in the considered state there are $\nu_{\vec{p}}$ particles with momentum $\vec{p}$ [25]. Single-particle energies are given as $\varepsilon_{\vec{p}}=\vec{p}^{2} / 2 m$ where $\vec{p}\left(p^{(x)}, p^{(y)}, p^{(z)}\right)$ is the eigenvalue of the momentum operator of a single particle

$$
p^{(a)}=\Delta p^{(a)} n^{(a)}, \quad n^{(a)}=0, \pm 1, \pm 2, \ldots, \quad a=x, y, z
$$

and $\Delta p^{(a)}$ is a step of the momentum grid towards the direction $a$ :

$$
\Delta p^{(a)}=\frac{2 \pi \hbar}{L}, \quad L=V^{1 / 3}
$$

In the second quantization method, Eq. (1) determines an infinite set of discrete values of momentum components of $\vec{p}$. Every discrete value of $\vec{p}$ corresponds to a single-particle state with energy $\varepsilon_{\vec{p}}$ and to a particle number $\nu_{\vec{p}}$. The total particle number $N$ and the total energy of this microscopic state $E_{\left\{\nu_{\vec{p}}\right\}}$ are

$$
\begin{gathered}
N=\sum_{\vec{p}} \nu_{\vec{p}} \\
E_{\left\{\nu_{\vec{p}}\right\} N}=\sum_{\vec{p}} \varepsilon_{\vec{p}} \nu_{\vec{p}} .
\end{gathered}
$$

The allowed occupation numbers are

$$
\begin{array}{ll}
\nu_{\vec{p}}=0,1,2, \ldots, N & \text { (for bosons) } \\
\nu_{\vec{p}}=0,1 & \text { (for fermions) } .
\end{array}
$$

The canonical ensemble partition function for the perfect Bose (Fermi) gas of $N$ identical particles is 25

$$
Q_{N}=\sum_{\left\{\nu_{\vec{p}}\right\}, \sum_{\vec{p}} \nu_{\vec{p}}=N} e^{-\beta E_{\left\{\nu_{\vec{p}}\right\} N}}
$$

where the total energy $E_{\left\{n_{\vec{p}}\right\}}$ is defined by Eq. (丰), the Lagrange multiplier $\beta(\equiv 1 / T)$ and the sum runs over all sets of occupation numbers (5), taking into account the conservation of the total particle number $N$. The expectation value of an operator $O$ over an ensemble of $N$ identical particles can be found in a standard way: 


$$
\langle O\rangle_{N}=\frac{1}{Q_{N}} \sum_{\left\{\nu_{\vec{p}}\right\}, \sum_{\vec{p}} \nu_{\vec{p}}=N} O_{\left\{\nu_{\vec{p}}\right\}} e^{-\beta E_{\left\{\nu_{\vec{p}}\right\} N} .}
$$

The partition function (6) can be treated as a generating function for moments of occupation number distributions. Then the average value (7) for different moments of occupation number distributions can be found in a following way. Let us introduce a new variable

$$
x_{\vec{p}}=e^{-\beta \varepsilon_{\vec{p}}}
$$

in the partition function (6)

$$
Q_{N}=\sum_{\left\{\nu_{\vec{p}}\right\}, \sum_{\vec{p}} \nu_{\vec{p}}=N} \prod_{\vec{p}} x_{\vec{p}}^{\nu_{\vec{p}}} .
$$

Then, the mean value and highest moments of occupation number distribution can be expressed through the derivatives of the partition function (9) with respect to $x_{\vec{p}}$

$$
\begin{aligned}
\left\langle\nu_{\vec{p}}\right\rangle_{N} & =\frac{1}{Q_{N}} x_{\vec{p}} \frac{\partial Q_{N}}{\partial x_{\vec{p}}} \\
\left\langle\nu_{\vec{p}} \nu_{\vec{p}^{\prime}}\right\rangle_{N} & =\frac{1}{Q_{N}} x_{\vec{p}} \frac{\partial}{\partial x_{\vec{p}}} x_{\vec{p}^{\prime}} \frac{\partial}{\partial x_{\vec{p}^{\prime}}} Q_{N} \\
\left\langle\nu_{\vec{p}} \nu_{\vec{p}^{\prime}} \nu_{\vec{p}^{\prime \prime}}\right\rangle_{N} & =\frac{1}{Q_{N}} x_{\vec{p}} \frac{\partial}{\partial x_{\vec{p}}} x_{\vec{p}^{\prime}} \frac{\partial}{\partial x_{\vec{p}^{\prime}}} x_{\vec{p}^{\prime \prime}} \frac{\partial}{\partial x_{\vec{p}^{\prime \prime}}} Q_{N} .
\end{aligned}
$$

The frequently used dispersion of the occupation numbers is

$$
\begin{aligned}
\Delta\left(\nu_{\vec{p}} \nu_{\vec{p}^{\prime}}\right)_{N} & =\left\langle\nu_{\vec{p}} \nu_{\vec{p}^{\prime}}\right\rangle_{N}-\left\langle\nu_{\vec{p}}\right\rangle_{N}\left\langle\nu_{\vec{p}^{\prime}}\right\rangle_{N} \\
& =x_{\vec{p}} \frac{\partial}{\partial x_{\vec{p}}} x_{\vec{p}^{\prime}} \frac{\partial}{\partial x_{\vec{p}^{\prime}}} \ln Q_{N} .
\end{aligned}
$$

Let us show now that the partition function $Q_{N}$ in the occupation number representation (6) is reduced to the multinomial sum [20,26] which can be solved by means of recursive equations. The comparative analysis of explicit expressions for the partition function $Q_{N}$ and the mean multiplicity $\left\langle\nu_{\vec{p}}\right\rangle_{N}$ for two systems having $N$ and $(N-1)$ particles reduces to the following relations (see Appendix A)

$$
\left\langle\nu_{\vec{p}}\right\rangle_{j} Q_{j}=e^{-\beta \varepsilon_{\vec{p}}} Q_{j-1}\left[1 \mp\left\langle\nu_{\vec{p}}\right\rangle_{j-1}\right], \quad j=1,2, \ldots, N
$$

with the initial values : $Q_{0}=1$ and $\left\langle\nu_{\vec{p}}\right\rangle_{0}=0$. The sign " +" " - " in Eq. (14) corresponds to the Bose-Einstein/Fermi-Dirac statistics, respectively. Eq. (14) is valid for both finite and

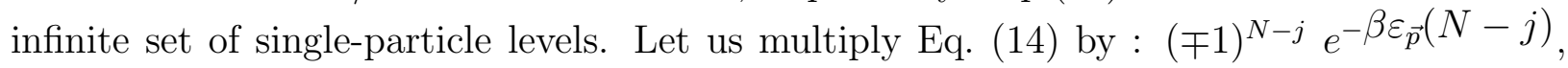
and sum both left hand side (l.h.s) and right hand side (r.h.s) of this equation over $j$ from 1 to $N$. This results in the following representation for the mean occupation numbers : 


$$
\begin{aligned}
\left\langle\nu_{\vec{p}}\right\rangle_{N} & =\frac{1}{Q_{N}} \sum_{j=1}^{N}(\mp 1)^{N-j} e^{-\beta(N-j+1) \varepsilon_{\vec{p}}} Q_{j-1} \\
& =\frac{1}{Q_{N}} \sum_{j=1}^{N}(\mp 1)^{j+1} e^{-\beta j \varepsilon_{\vec{p}}} Q_{N-j} .
\end{aligned}
$$

By the substitution of (15) into the total particle number conservation condition (3), we arrive at the related set of recurrence equations for the partition functions (6) of systems with different number of particles [20]

$$
Q_{N}=\frac{1}{N} \sum_{j=1}^{N} f_{j} Q_{N-j}, \quad Q_{0}=1,
$$

where

$$
f_{j}=(\mp 1)^{j+1} \sum_{\vec{p}} e^{-\beta j \varepsilon_{\vec{p}}}
$$

This solves the problem of finding the canonical ensemble partition function. The sum in (17) runs over the whole infinite set of eigenvalues of the momentum operator of a single particle. Inserting different $Q_{j}$ 's into the recurrence relations (16) gives rise to the multinomial sum

$$
Q_{N}=\sum_{\left\{n_{j}\right\}, \sum_{j=1}^{N} j n_{j}=N} \prod_{l=1}^{N} \frac{f_{l}^{n_{l}}}{l^{n_{l}} n_{l} !}
$$

which is the well-known quantum-group Meyer expansion for the ideal Bose (Fermi) gas of identical particles in the canonical ensemble method $\left(n_{j}\right.$ are integer numbers) [25]. One should note that the recurrence relations (16) are satisfied for any multinomial sum (18), independently of an explicit form of the $f_{l}$ variable [20]. The recurrence relations for the case of the ideal gas of $N$ identical fermions were obtained recently by Pratt [27] using of somewhat different technique.

The mean occupation number is given by Eq. (15). To get the second moment of the occupation number distributions, let us compare the r.h.s. of Eqs. (15) and (10). Using the definition (8) of $x_{\vec{p}}$ variable, we find the following relation between the first derivative of the partition function $Q_{N}$ with respect to $x_{\vec{p}}$ and the partition functions of systems with smaller number of particles

$$
x_{\vec{p}} \frac{\partial Q_{N}}{\partial x_{\vec{p}}}=\sum_{j=1}^{N}(\mp 1)^{j+1} x_{\vec{p}}^{j} Q_{N-j} .
$$

Substituting (19) into Eq. (11) and using again (8), we obtain finally

$$
\begin{aligned}
& \left\langle\nu_{\vec{p}} \nu_{\vec{p}^{\prime}}\right\rangle_{N}=\frac{1}{Q_{N}} \sum_{i=1}^{N} \sum_{j=1}^{N-i}(\mp 1)^{i+j} e^{-\beta i \varepsilon_{\vec{p}}} e^{-\beta j \varepsilon_{\vec{p}^{\prime}}} Q_{N-i-j} \\
& +\delta_{\vec{p} \vec{p}^{\prime}} \frac{1}{Q_{N}} \sum_{j=1}^{N}(\mp 1)^{j+1} e^{-\beta j \varepsilon_{\vec{p}}} j Q_{N-j} .
\end{aligned}
$$


The knowledge of the mean occupation numbers (15), their second moments (20) or dispersions (13) is sufficient for evaluating most of the thermodynamic quantities. Higher moments of occupation numbers can be found, if necessary, by the same procedure as presented above.

Similar results can be obtained for the limiting case of an ideal gas of classical Boltzmann particles in the canonical ensemble. One should take into account the "correct Boltzmann counting" [25], i.e., that for an arbitrary set of occupations numbers satisfying (3) there are generally more ways to construct an ensemble as compared to the Bose gas because the interchange of any distinguishable particles leaves the set $\left\{\nu_{\vec{p}}\right\}$ unchanged

$$
Q_{N}^{B}=\frac{1}{N !} \sum_{\left\{\nu_{\vec{p}}\right\}, \sum_{\vec{p}} \nu_{\vec{p}}=N} \frac{N !}{\prod_{\vec{p}} \nu_{\vec{p}} !} e^{-\beta E}\left\{\nu_{\vec{p}}\right\} N
$$

where the total energy $E_{\left\{\nu_{\vec{p}}\right\}}$ is given by Eq. (4) and the sum runs over all allowed values of occupation numbers : $\nu_{\vec{p}}=0,1, \ldots, N$, taking into account the conservation of the total particle number (3). This sum is the multinomial Newton sum which can be calculated exactly [25]

$$
Q_{N}^{B}=\frac{1}{N !}\left(\sum_{\vec{p}} e^{-\beta \varepsilon_{\vec{p}}}\right)^{N} .
$$

The partition function for the ideal Boltzmann gas (22) corresponds to a single term of the multinomial sum (18) of quantum Bose gas with $n_{1}=N$ and $n_{j}=0$ for $j \neq 1$

$$
Q_{N}^{B}=\frac{\omega^{N}}{N !}, \quad \omega \equiv f_{1}=\sum_{\vec{p}} e^{-\beta \varepsilon_{\vec{p}}} .
$$

Therefore, all results presented above for the Bose gas and expressed through functions $f_{j}$ and $e^{-\beta j \varepsilon_{\vec{p}}}$, can be immediately applied to the ideal Boltzmann gas after a simple substitution

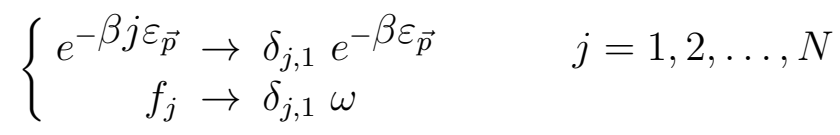

where $\delta_{j, 1}$ is the Kronecker symbol. Thus, in the limiting case of the classical Boltzmann statistics only the term with $j=1$ survives in each sum over $j$ in Eqs. (15)-(17), (19), (20). For example, inserting (24) into Eqs. (16) results in the following recurrence equations for an ideal Boltzmann gas

$$
Q_{N}^{B}=\frac{1}{N} \omega Q_{N-1}^{B}
$$

from where directly follows Eq. (23). Using (24), (23) and (25) we have for the mean occupation number

$$
\left\langle\nu_{\vec{p}}\right\rangle_{N}^{B}=e^{-\beta \varepsilon_{\vec{p}}} \frac{Q_{N-1}^{B}}{Q_{N}^{B}}=N \frac{e^{-\beta \varepsilon_{\vec{p}}}}{\sum_{\vec{p}} e^{-\beta \varepsilon_{\vec{p}}}} .
$$


Analogously, the second momentum of the occupation number distribution reduces to the form

$$
\left\langle\nu_{\vec{p}} \nu_{\vec{p}^{\prime}}\right\rangle_{N}^{B}=N(N-1) \frac{e^{-\beta \varepsilon_{\vec{p}}} e^{-\beta \varepsilon_{\vec{p}^{\prime}}}}{\left(\sum_{\vec{p}} e^{-\beta \varepsilon_{\vec{p}}}\right)^{2}}+\delta_{\vec{p} \vec{p}^{\prime}} N \frac{e^{-\beta \varepsilon_{\vec{p}}}}{\sum_{\vec{p}} e^{-\beta \varepsilon_{\vec{p}}}}
$$

which is well-known in statistical mechanics [25].

Let us now consider fluctuation of particle number distributions over different quantum states. The mean squared occupation numbers $\left\langle\nu_{\vec{p}}^{2}\right\rangle_{N}$ for Bose and Fermi statistics are given in Eq. (20) which can be rewritten as follows

$$
\left\langle\nu_{\vec{p}}^{2}\right\rangle_{N}=\frac{1}{Q_{N}} \sum_{j=1}^{N}(\mp 1)^{j+1} e^{-\beta j \varepsilon_{\vec{p}}} Q_{N-j}[\mp(j-1)+j] .
$$

Using Eqs. (15) and (28) we get for the occupation number dispersion in the Fermi gas

$$
\left\langle\left(\Delta \nu_{\vec{p}}\right)^{2}\right\rangle_{N} \equiv\left\langle\left(\nu_{\vec{p}}-\left\langle\nu_{\vec{p}}\right\rangle_{N}\right)^{2}\right\rangle_{N}=\left\langle\nu_{\vec{p}}\right\rangle_{N}\left[1-\left\langle\nu_{\vec{p}}\right\rangle_{N}\right]
$$

This expression coincides exactly with the appropriate expression calculated within the grand canonical method [28]. This is due to the equality : $\left\langle\nu_{\vec{p}}^{2}\right\rangle_{N}=\left\langle\nu_{\vec{p}}\right\rangle_{N}$, which follows from (15) and (28).

Similar consideration for the Bose gas gives

$$
\left\langle\left(\Delta \nu_{\vec{p}}\right)^{2}\right\rangle_{N}=\frac{1}{Q_{N}} \sum_{j=1}^{N}(\mp 1)^{j+1} e^{-\beta j \varepsilon_{\vec{p}}}(2 j-1) Q_{N-j}-\left\langle\nu_{\vec{p}}\right\rangle_{N}^{2} .
$$

For the case of the Boltzmann gas we have from Eqs. (26) and (27)

$$
\left\langle\left(\Delta \nu_{\vec{p}}\right)^{2}\right\rangle_{N}^{B}=\left\langle\nu_{\vec{p}}\right\rangle_{N}^{B}\left[1-\frac{\left\langle\nu_{\vec{p}}\right\rangle_{N}^{B}}{N}\right]
$$

Here the first term corresponds to the grand canonical result $[28]:\left\langle\left(\Delta \nu_{\vec{p}}\right)^{2}\right\rangle_{G C}=\left\langle\nu_{\vec{p}}\right\rangle_{G C}$, where $\langle\ldots\rangle_{G C}$ denotes the grand canonical ensemble averaging. As is seen, in the canonical ensemble treatment an additional negative term arises which vanishes in the thermodynamic limit $N \rightarrow \infty$.

Let us introduce the quantity $\eta$ as an integral measure of correlations between the occupation numbers 28

$$
\eta=\frac{\sum_{\vec{p}}\left[\left\langle\left(\Delta \nu_{\vec{p}}\right)^{2}\right\rangle_{N}-\left\langle\nu_{\vec{p}}\right\rangle_{N}\right]}{\sum_{\vec{p}}\left\langle\nu_{\vec{p}}\right\rangle_{N}}
$$

In application to the Fermi gas, using Eq. (29), we have

$$
\eta=-\frac{1}{N} \sum_{\vec{p}}\left\langle\nu_{\vec{p}}\right\rangle_{N}^{2}
$$


It is seen that $\eta$ is negative for all values of $T, V$ and $\eta=-1$ at the limiting temperature $T=$ 0 . In the grand canonical ensemble for the Fermi gas we have : $\eta=-\left(1 /\langle N\rangle_{G C}\right) \sum_{\vec{p}}\left\langle\nu_{\vec{p}}\right\rangle_{G C}^{2}$. For the correlation of identical particles in the Bose gas, substituting (30) into (32) and using (15), we have

$$
\eta=\frac{1}{N}\left\{\frac{1}{Q_{N}} \sum_{j=1}^{N} f_{j} 2(j-1) Q_{N-j}-\sum_{\vec{p}}\left\langle\nu_{\vec{p}}\right\rangle_{N}^{2}\right\} .
$$

At $T=0$ one finds : $\eta=-1$, because : $Q_{N}=1$ and $f_{j}=1$ for any $N$ and $j$. In the grand canonical ensemble : $\eta=+\left(1 /\langle N\rangle_{G C}\right) \sum_{\vec{p}}\left\langle\nu_{\vec{p}}\right\rangle_{G C}^{2}$. Similarly, in the Boltzmann limit of the Bose gas with using Eq. (32), we have :

$$
\eta^{B}=-\frac{1}{N^{2}} \sum_{\vec{p}}\left\langle\nu_{\vec{p}}\right\rangle_{N}^{2}
$$

In this case the grand canonical method yields $\eta=0$. At $T=0$ one finds $: \eta=-1$, because the mean occupation numbers (26) are equal to : $\left\langle\nu_{\vec{p}}\right\rangle_{N}=N \delta_{\vec{p}, 0}$.

Thus, the occupation number dispersion in the canonical ensemble for Bose and Boltzmann statistics differs from those in the grand canonical, because the statistical independence of a particle in a state $\vec{p}$ is broken due to the requirement of the total particle number conservation (3). At the same time, all three statistics predict for the value of the correlation coefficient $\eta \rightarrow-1$ when $T \rightarrow 0$.

\section{IDEAL GAS MIXTURE OF BOSE-FERMI FRAGMENTS}

Let us consider a system consisting of $N_{1}$ individual nucleons, $N_{2}$ clusters (fragments) made of two nucleons and so on until $N_{A}$ fragments of $A$ nucleons, contained in the volume $V$ at temperature $T$. The composition of this mixture is characterized by a given set of fragment numbers $\left(N_{1}, N_{2}, \ldots, N_{A}\right)$ and satisfies the nucleon number conservation condition

$$
\sum_{i=1}^{A} i N_{i}=A
$$

A fragment of any species $i$ follows either Bose-Einstein or Fermi-Dirac statistics and a number of fragments of a given species $N_{i}$ is assumed to be conserved at all permutations. Let us derive the thermodynamic relations for such a mixture of ideal Bose and Fermi fragments within the canonical ensemble method.

A microscopic state of an ideal Bose or Fermi gas subsystem of $i$-th species is defined by a set of occupation numbers $\left\{\nu_{\vec{p} i}\right\}$ for single-particle levels of the fragment $i$. This implies that the microscopic state in question has $\nu_{\vec{p} i}$ clusters of mass $i$ with momentum $\vec{p}$ [25]. The single-particle energies are : $\varepsilon_{\vec{p} i}=\vec{p}^{2} / 2 m_{i}$.

The fragment number $N_{i}$ of every species $i$ and the total energy of quantum gas mixture of a given partition or composition : $E_{\left\{\nu_{\vec{p} i}\right\}} N_{1}, N_{2}, \ldots, N_{A}$, are defined by the following expressions (cp. to (3) and (4) ) : 


$$
\begin{aligned}
N_{i} & =\sum_{\vec{p}} \nu_{\vec{p} i}, \quad i=1,2, \ldots, A \\
E_{\left\{\nu_{\vec{p} i}\right\} N_{1}, N_{2}, \ldots, N_{A}} & =\sum_{i, \vec{p}} \varepsilon_{\vec{p} i} \nu_{\vec{p} i}
\end{aligned}
$$

with the allowed values of occupation numbers given in (5)).

Generalization of the canonical ensemble partition function (6) to the case of an ideal gas mixture of non-identical Bose-Fermi particles with the conservation of a number of fragments of every species (37) can be written as [25]

$$
W_{A}\left(N_{1}, \ldots, N_{A}\right)=\sum_{\left\{\nu_{\vec{p} \imath}\right\}} e^{-\beta E_{\left\{\nu_{\vec{p} i}\right\} N_{1}, \ldots, N_{A}}}
$$

where the sum runs over all partitions of occupation numbers (5)

$$
\sum_{\left\{\nu_{\vec{p} i}\right\}} \equiv \sum_{\left\{\nu_{\vec{p} 1}\right\}\left\{\nu_{\vec{p} 2}\right\} \ldots,\left\{\nu_{\vec{p} A}\right\}} \prod_{i=1}^{A} \delta\left(\sum_{\vec{p}} \nu_{\vec{p} i}-N_{i}\right)
$$

satisfying the conservation laws (37). Generalization of ensemble averaging (77) to the case of an ideal gas of Bose-Fermi fragments of different masses is

$$
\langle O\rangle_{N_{1}, \ldots, N_{A}}=\frac{1}{W_{A}\left(N_{1}, \ldots, N_{A}\right)} \sum_{\left\{\nu_{\vec{p}}\right\}} O\left(N_{1}, \ldots, N_{A}\right) e^{\left.-\beta E_{\left\{\nu_{\vec{p}}\right\}}\right\} N_{1}, \ldots, N_{A}} .
$$

Since fragments do not interact with each other, the total partition function (39) is reduced to the product of partition functions $Q\left(N_{i}\right)$ for subsystems of identical fragments $N_{i}$

$$
W_{A}\left(N_{1}, \ldots, N_{A}\right)=\prod_{i=1}^{A} Q\left(N_{i}\right) .
$$

Evaluation of the partition function $Q\left(N_{i}\right)$ for a subsystem of $N_{i}$ identical Bose (Fermi) fragments of the mass $i$ has been considered in the previous section. Then, Eqs. (6), (16) and (17) can be rewritten in the new notation as follows

$$
\begin{aligned}
Q\left(N_{i}\right) & =\sum_{\left\{\nu_{\vec{p} \imath}\right\}, \sum_{\vec{p}} \nu_{\vec{p} i}=N_{i}} \prod_{\vec{p}} e^{-\beta \varepsilon_{\vec{p} i} \nu_{\vec{p} \imath}} \\
& =\frac{1}{N_{i}} \sum_{l=1}^{N_{i}} f_{i l} Q\left(N_{i}-l\right), \quad i=1,2, \ldots, A \\
f_{i l} & =(\mp 1)^{l+1} \sum_{\vec{p}} e^{-\beta l \varepsilon_{\vec{p} i}}
\end{aligned}
$$

where the signs $(+)$ and $(-)$ correspond to the Bose-Einstein and Fermi-Dirac statistics, respectively.

In the same manner, the mean occupation numbers and their second momenta for a subsystem of $N_{i}$ ideal Bose (Fermi) identical fragments of the species $i$ can be expressed by 
the canonical ensemble partition function (43) for smaller number of fragments of the same species (see (15), (20) )

$$
\begin{aligned}
\left\langle\nu_{\vec{p} i}\right\rangle_{N_{i}} & =\frac{1}{Q\left(N_{i}\right)} \sum_{l=1}^{N_{i}}(\mp 1)^{l+1} e^{-\beta l \varepsilon_{\vec{p} i}} Q\left(N_{i}-l\right) \\
\left\langle\nu_{\vec{p} i} \nu_{\vec{p}^{\prime} i}\right\rangle_{N_{i}} & =\frac{1}{Q\left(N_{i}\right)} \sum_{l=1}^{N_{i}} \sum_{k=1}^{N_{i}-l}(\mp 1)^{l+k} e^{-\beta l \varepsilon_{\vec{p} \imath}} e^{-\beta k \varepsilon_{\vec{p}^{\prime} i}} Q\left(N_{i}-l-k\right) \\
& +\delta_{\vec{p} \vec{p}^{\prime}} \frac{1}{Q\left(N_{i}\right)} \sum_{l=1}^{N_{i}}(\mp 1)^{l+1} e^{-\beta l \varepsilon_{\vec{p} i}} l Q\left(N_{i}-l\right) .
\end{aligned}
$$

By the subsequent substitution of the recurrence relations (44) into Eq. (42), the partition of an ideal quantum gas mixture is reduced to the generalized quantum-group Meyer expansion (cp. to Eq. (18))

$$
W_{A}\left(N_{1}, \ldots, N_{A}\right)=\prod_{i=1}^{A} \sum_{\left\{n_{j}\right\}, \sum_{j} j n_{j}=N_{i}} \prod_{l=1}^{N_{i}} \frac{\left(f_{i l}\right)^{n_{l}}}{l^{n_{l}} n_{l} !}
$$

Comparing the definitions of ensemble averaging for identical particles (7) to that for a mixture of Bose-Fermi fragments (41), we can relate the first and second moments of occupation numbers as well as their dispersions, to appropriate quantities for subsystems of identical fragments:

$$
\begin{aligned}
& \left\langle\nu_{\vec{p} i}\right\rangle_{N_{1}, \ldots, N_{A}}=\left\langle\nu_{\vec{p} i}\right\rangle_{N_{i}} \\
& \left\langle\nu_{\vec{p} i} \nu_{\vec{p}^{\prime} j}\right\rangle_{N_{1}, \ldots, N_{A}}=\delta_{i j}\left\langle\nu_{\vec{p} \imath} \nu_{\vec{p}^{\prime} j}\right\rangle_{N_{i}}+\left(1-\delta_{i j}\right)\left\langle\nu_{\vec{p} \imath}\right\rangle_{N_{i}}\left\langle\nu_{\vec{p}^{\prime} j}\right\rangle_{N_{j}} \\
& \Delta\left(\nu_{\vec{p} i} \nu_{\vec{p}^{\prime} j}\right)_{N_{1}, \ldots, N_{A}}=\left\langle\nu_{\vec{p} i} \nu_{\vec{p}^{\prime} j}\right\rangle_{N_{1}, \ldots, N_{A}}-\left\langle\nu_{\vec{p} i}\right\rangle_{N_{1}, \ldots, N_{A}}\left\langle\nu_{\vec{p}^{\prime} j}\right\rangle_{N_{1}, \ldots, N_{A}} .
\end{aligned}
$$

Therefore, to find the moments of occupation number distributions (49)-(51) for a mixture of quantum gas with fixed composition of fragments, it is sufficient to know the occupation number moments for subsystems of $N_{i}$ identical fragments. The latter are related via Eqs. (46), (47) to the subsystem partition function $Q\left(N_{i}\right)$ and can be calculated recursively by means of Eq. (44).

Let us turn now to the calculation of general quantities characterizing thermodynamic behavior of a system. By definition, the average energy of an ideal gas mixture of Bose-Fermi non-identical fragments is a partial derivative of the logarithm of the partition function (39) with respect to the temperature at a constant volume $V$

$$
E\left(N_{1}, \ldots, N_{A}\right)=\left.T^{2} \frac{\partial \ln W_{A}\left(N_{1}, \ldots, N_{A}\right)}{\partial T}\right|_{V}=\left\langle E_{\left\{N_{\vec{p}}\right\}, N_{1}, \ldots, N_{A}}\right\rangle_{N_{1}, \ldots, N_{A}}
$$

where the total energy expectation value is calculated over the ensemble (41) and the sum runs over all values of the occupation numbers (5). Because the gas is ideal, the average energy of the system is the sum of subsystem energies

$$
E\left(N_{1}, \ldots, N_{A}\right)=\sum_{i, \vec{p}} \varepsilon_{\vec{p} i}\left\langle\nu_{\vec{p} \imath}\right\rangle_{N_{1}, \ldots, N_{A}} .
$$


Inserting the mean occupation numbers (49), expressed through the partition functions for subsystems of identical Bose-Fermi fragments (46), one obtains

$$
\begin{aligned}
E\left(N_{1}, \ldots, N_{A}\right) & =\sum_{i=1}^{A} \frac{1}{Q\left(N_{i}\right)} \sum_{l=1}^{N_{i}} \overline{\varepsilon_{i l}} Q\left(N_{i}-l\right) \\
\overline{\varepsilon_{i l}} & =(\mp 1)^{l+1} \sum_{\vec{p}} \varepsilon_{\vec{p} i} e^{-\beta l \varepsilon_{\vec{p} i}} .
\end{aligned}
$$

The summation procedure over the fragment momentum $\vec{p}$ in Eq. (55) is presented in the Appendix B.

By the definition, the pressure in the system is

$$
P\left(N_{1}, \ldots, N_{A}\right)=\left.T \frac{\partial \ln W_{A}\left(N_{1}, \ldots, N_{A}\right)}{\partial V}\right|_{T}=-\left\langle\frac{\partial E_{\left\{N_{\vec{p} i}\right\}, N_{1}, N_{2}, \ldots, N_{A}}}{\partial V}\right\rangle_{N_{1}, \ldots, N_{A}}
$$

Inserting here the average energy (38), we find

$$
P\left(N_{1}, \ldots, N_{A}\right)=-\sum_{i, \vec{p}} \frac{\partial \varepsilon_{\vec{p} i}}{\partial V}\left\langle\nu_{\vec{p} i}\right\rangle_{N_{1}, \ldots, N_{A}}
$$

Making use of Eqs. (46), (49), the pressure in a mixed system (57) can be related to the partition functions for the Bose-Fermi subsystems of identical fragments (44)

$$
\begin{aligned}
P\left(N_{1}, \ldots, N_{A}\right) & =\sum_{i=1}^{A} \frac{1}{Q\left(N_{i}\right)} \sum_{l=1}^{N_{i}} \overline{P_{i l}} Q\left(N_{i}-l\right) \\
\overline{P_{i l}} & =-(\mp 1)^{l+1} \sum_{\vec{p}} \frac{\partial \varepsilon_{\vec{p} i}}{\partial V} e^{-\beta l \varepsilon_{\vec{p} i}} .
\end{aligned}
$$

In a similar way, one can calculate the specific heat of the system which is defined as a derivative of the average energy (52) with respect to temperature at the constant volume

$$
\begin{aligned}
C_{V}\left(N_{1}, \ldots, N_{A}\right) & =\left.\frac{\partial E\left(N_{1}, \ldots, N_{A}\right)}{\partial T}\right|_{V} \\
& =\frac{\left.\left\langle E_{\left\{\nu_{\vec{p}}\right\}}^{2}\right\}, N_{1}, \ldots, N_{A}\right\rangle_{N_{1}, \ldots, N_{A}}-\left\langle E_{\left.\left\{\nu_{\vec{p}}\right\}, N_{1}, \ldots, N_{A}\right\rangle_{N_{1}, \ldots, N_{A}}^{2}}\right.}{T^{2}} .
\end{aligned}
$$

Inserting (38) into (41) and using (49)-(51), we express the specific heat for mixture of an ideal quantum gas as

$$
C_{V}\left(N_{1}, \ldots, N_{A}\right)=\beta^{2} \sum_{i, j, \vec{p}, \vec{p}^{\prime}} \Delta\left(N_{\vec{p} i} \nu_{\vec{p}^{\prime} j}\right)_{N_{1}, \ldots, N_{A}} \varepsilon_{\vec{p} i} \varepsilon_{\vec{p}^{\prime} j}
$$

Hence, the specific heat can be expressed via the partition functions of identical Bose-Fermi fragment subsystems (43), if one applies Eqs. (46), (47) and (49), (50)

$$
\begin{aligned}
C_{V}\left(N_{1}, \ldots, N_{A}\right)= & \beta^{2} \sum_{i=1}^{A} \frac{1}{Q\left(N_{i}\right)} \sum_{l, k} \overline{\varepsilon_{i l}} \overline{\varepsilon_{i k}}\left[Q\left(N_{i}-l-k\right)-\frac{1}{Q\left(N_{i}\right)} Q\left(N_{i}-l\right) Q\left(N_{i}-k\right)\right] \\
& +\beta^{2} \sum_{i=1}^{A} \frac{1}{Q\left(N_{i}\right)} \sum_{l=1}^{N_{i}} l \overline{\varepsilon_{i l}^{2}} Q\left(N_{i}-l\right) .
\end{aligned}
$$


In the above equations, the quantity $\overline{\varepsilon_{i l}}$ is defined by Eq. (55) and the average squared energy of a fragment is

$$
\overline{\varepsilon_{i l}^{2}}=(\mp 1)^{l+1} \sum_{\vec{p}} \varepsilon_{\vec{p} \imath}^{2} e^{-\beta l \varepsilon_{\vec{p} \imath}}
$$

Evaluation of average values of $\overline{\varepsilon_{i l}}$ and $\overline{\varepsilon_{i l}^{2}}$ is given in the Appendix B.

Let us consider now the Boltzmann limit of an ideal gas mixture of Bose-Fermi fragments. The partition function of the ideal Boltzmann gas of non-identical fragments taking into account the conservation law for fragment numbers of every species (37) is (cp. to (22)) :

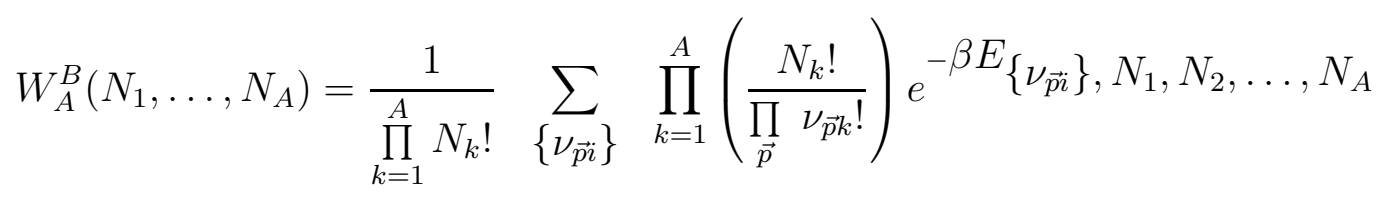

where total energy $E_{\left\{\nu_{\vec{p}}\right\}, N_{1}, N_{2}, \ldots, N_{A}}$ is given again by Eq. (38) and occupation numbers take values : $\nu_{\vec{p} i}=0,1, \ldots, N_{i}$. Similarly to the quantum partition function (39), the classical partition function (64) can be expressed as a product of partition functions $Q^{B}\left(N_{i}\right)$ for subsystems of $N_{i}$ identical Boltzmann fragments of mass $i$. As was shown in the preceding Section, the partition function $Q^{B}\left(N_{i}\right)$ for the subsystem of $N_{i}$ Boltzmann identical fragments of mass $i$, which is obtained from the appropriate quantum equation by the simple substitution of (24)

$$
Q^{B}\left(N_{i}\right)=\frac{\omega_{i}^{N_{i}}}{N_{i} !}, \quad \omega_{i} \equiv f_{i 1}=\sum_{\vec{p}} e^{-\beta \varepsilon_{\vec{p} \imath}}
$$

corresponds to a single term of the multinomial sum (18) with $n_{i 1}=N_{i}$ and $n_{i j}=0$ for $j \neq 1(i=1,2, \ldots, A)$ 25]. So, the substitution of (24) into the recurrence relations (44) leaves only one term

$$
Q^{B}\left(N_{i}\right)=\frac{1}{N_{i}} \omega_{i} Q^{B}\left(N_{i}-1\right), \quad i=1,2, \ldots, A
$$

and the partition function (48) for the mixed Boltzmann gas of fragments takes very simple form:

$$
W_{A}^{B}\left(N_{1}, \ldots, N_{A}\right)=\prod_{i=1}^{A} \frac{\omega_{i}^{N_{i}}}{N_{i} !}
$$

Eqs. (49)-(51) for the moments of the occupation numbers remains unchanged but the evaluation of the corresponding momentum components is simplified noticeably (see (26), (27)). Taking into account Eqs. (55) and (65) for the average energy (54), we have

$$
E^{B}\left(N_{1}, \ldots, N_{A}\right)=\sum_{i=1}^{A} \overline{\varepsilon_{i}} N_{i}
$$

where 


$$
\bar{\varepsilon}_{i}=\frac{\sum_{\vec{p}} \varepsilon_{\vec{p} i} e^{-\beta \varepsilon_{\vec{p} i}}}{\sum_{\vec{p}} e^{-\beta \varepsilon_{\vec{p} i}}} .
$$

From Eqs. (58), (59) and (65), one finds that the pressure in a mixture of the ideal Boltzmann gas is

$$
P^{B}\left(N_{1}, \ldots, N_{A}\right)=\sum_{i=1}^{A} \overline{P_{i}} N_{i}
$$

where

$$
\overline{P_{i}}=-\frac{\sum_{\vec{p}} \frac{\partial \varepsilon_{\vec{p} i}}{\partial V} e^{-\beta \varepsilon_{\vec{p} i}}}{\sum_{\vec{p}} e^{-\beta \varepsilon_{\vec{p} i}}}
$$

Specific heat of the system in the Boltzmann limit is obtained from Eqs. (62), (63), (55) and (65)

$$
C_{V}^{B}\left(N_{1}, \ldots, N_{A}\right)=\beta^{2} \sum_{i=1}^{A}\left(\overline{\varepsilon_{i}^{2}}-\bar{\varepsilon}_{i}^{2}\right) N_{i}
$$

with

$$
\overline{\varepsilon_{i}^{2}}=\frac{\sum_{\vec{p}} \varepsilon_{\vec{p} i}^{2} e^{-\beta \varepsilon_{\vec{p} i}}}{\sum_{\vec{p}} e^{-\beta \varepsilon_{\vec{p} i}}} .
$$

These relations will be used below when describing the thermodynamics of the quantum statistical model of the multifragmentation.

\section{QUANTUM STATISTICAL MODEL OF NUCLEAR MULTIFRAGMENTATION}

\section{A. Model formulation}

Based on the recurrence technique discussed above, we shall formulate now a quantum model of fragmentation of a finite nuclear system into nucleons and nucleon clusters. This quantum fragmentation model is constructed in the the framework of the canonical ensemble description in the occupation number representation. Along this way, we shall closely follow the physical approximations used in the popular statistical fragmentation models in the canonical ensemble [6,29, 30]. In particular, the partition function of a fragmenting system is defined as a sum over all fragment partitioning, each terms of which is taken with the weight associated with the partition function of a given fragment composition. 
The problem of the partitioning of an integer $A$ into a sum of integer numbers is known in the number theory for a long time [20,26]. Some multifragmentation models 31, 32 even employed this mathematical result for the description of mass fragment distributions assuming equal probability for any fragment composition. A partition of $A$ nucleons into clusters $\left(N_{1}, \ldots, N_{A}\right)$ corresponds to the fragmentation of a nucleus $A$ into $N_{1}$ individual nucleons, $N_{2}$ fragments made of 2 nucleons etc. with the nucleon conservation constraint (36). To construct a multifragmentation model, it is necessary to define a probability of any partition $\left(N_{1}, \ldots, N_{A}\right)$ with the natural condition that the sum of partial probabilities over all final states equals one. In the statistical multifragmentation models [6], the nonnormalized probability of a fragment configuration is given by its partition function. This is just the quantity $W_{A}\left(N_{1}, N_{2}, \ldots, N_{A}\right)$ studied in Sec. 3 .

Strictly speaking, the ideal gas approximation does not address the problem of the nuclear multifragmentation process properly, especially with respect to a possible relation of multifragmentation to the liquid-gas phase transition. The physics content of the model can be however improved by noting that the decisive condition allowing to develop the general scheme of recurrence equations is the additivity of the total system energy with respect to subsystem contributions. So, the interaction of $i$ nucleons bound in a single cluster can be taken into account by adding the fragment binding energy $b_{i}$ to the single particle energy:

$$
\varepsilon_{\vec{p} i}=\frac{\vec{p}^{2}}{2 m_{i}}+b_{i}
$$

In principle, some mean-field interaction energy depending only on $i$ can be also included into Eq. (74). In particular, such a form has the Coulomb interaction energy between fragments in the Wigner-Seitz approximation

$$
E_{i}^{W-S}=\frac{3}{5} \frac{A^{2} e^{2}}{4 R}\left(\frac{i}{A}\right)\left[1-\left(\frac{i}{A}\right)^{2 / 3}\right], \quad R=\left(\frac{3 V}{4 \pi}\right)^{1 / 3}
$$

which is used frequently in the statistical description of multifragmentation [6,29,30].

Repulsive residual nuclear interaction between fragments can be approximately accounted for in equilibrium statistical mechanics by the excluded volume method [25, 33. Since a real fragment occupies some finite volume $v_{i}$, therefore only a reduced volume is reachable for their free motion:

$$
V_{f}=V-\sum_{i=1}^{A} v_{i} N_{i}
$$

It is then assumed that the system may be still treated as consisting of idealized point-like fragments in $V_{f}$. It is worth noting that a simple replacement in thermodynamic equations : $V \rightarrow V_{f}$, needs certain care. Appearance of an additional $N_{i}$ dependence of the partition function may result in the loss of thermodynamic self-consistency. Due to the lack of consistency some basic relations of thermodynamics, such as those relating a derivative of the thermodynamic potential to the particle number or the pressure, may be violated [34 36]. Moreover, as suggested by (76), the multiplicity dependence influences the probability of a given fragment partition which is proportional to the (micro)canonical weight of a given partition and therefore should be properly accounted for. Recent discussions of this problem 
can be found in Refs. 37 40. In the QSM, the excluded volume effect is ignored and $V$ is treated as a free variable parameter.

Thus, the canonical ensemble partition function in the QSM of multifragmentation can be written as follows:

$$
\begin{aligned}
Z_{A} & =\sum_{\left\{N_{j}\right\}, \sum_{j} j N_{j}=A} W_{A}\left(N_{1}, \ldots, N_{A}\right) \\
& =\sum_{\left\{N_{j}\right\}, \sum_{j} j N_{j}=A} \sum_{\left\{\nu_{\vec{p} i}\right\}} e^{-\beta E_{\left\{\nu_{\vec{p} i}\right\} N_{1}, \ldots, N_{A}}}
\end{aligned}
$$

where the sum runs over all possible fragment partitioning $\left(N_{1}, N_{2}, \ldots, N_{A}\right)$ subject to the constraint (36). The partition function for a given fragment configuration is described by Eq. (39) together with the conservation laws (37) for each fragment $i$. Because the partition function $W_{A}\left(N_{1}, \ldots, N_{A}\right)$ can be expressed through the multinomial sum (48) depending on variables (45), we have

$$
Z_{A}=\sum_{\left\{N_{j}\right\}, \sum_{j} j N_{j}=A} \prod_{i=1}^{A} \sum_{\left\{n_{k}\right\}, \sum_{k} k n_{k}=N_{i}} \prod_{l=1}^{N_{i}} \frac{\left(f_{i l}\right)^{n_{l}}}{l^{n_{l}} n_{l} !} .
$$

The expectation value of a given operator $O$ over full ensemble of a system decaying into ideal Bose and Fermi fragments is defined in the QSM as follows (cp. to (41)) :

$$
\begin{aligned}
\langle O\rangle_{A} & =\frac{1}{Z_{A}} \sum_{\left\{N_{j}\right\}, \sum_{j} j N_{j}=A}\langle O\rangle_{N_{1}, \ldots, N_{A}} W_{A}\left(N_{1}, \ldots, N_{A}\right) \\
& =\frac{1}{Z_{A}} \sum_{\left\{N_{j}\right\}, \sum_{j} j N_{j}=A} \sum_{\left\{\nu_{\vec{p} i}\right\}} O\left\{\nu_{\vec{p} i}\right\} e^{\left.-\beta E_{\left\{\nu_{\vec{p}}\right\}}\right\} N_{1}, \ldots, N_{A} .}
\end{aligned}
$$

Let us find the recurrence relations for $Z_{A}$. According to (79), the average occupation numbers can be rewritten in the following form

$$
\left\langle\nu_{\vec{p} \imath}\right\rangle_{A}=\frac{1}{Z_{A}} \sum_{\left\{N_{j}\right\}, \sum_{j} j N_{j}=A}\left\langle\nu_{\vec{p} i}\right\rangle_{N_{1}, \ldots, N_{A}} \prod_{l=1}^{A} Q\left(N_{l}\right), \quad i=1,2, \ldots, A
$$

where Eq. (42) was used for $W_{A}\left(N_{1}, \ldots, N_{A}\right)$. The insertion of (49) and then (46) into Eq. (80) and the exchange of the summation order over $l$ and $\left\{N_{j}\right\}$ followed by the extension of the sum over $l$ to the maximal value $N_{i}$ by means of the step-function $\theta\left(N_{i}-l\right)$, gives

$$
\left\langle\nu_{\vec{p} i}\right\rangle_{A}=\frac{1}{Z_{A}} \sum_{l=1}^{[A / i]}(\mp 1)^{l+1} e^{-\beta l \varepsilon_{\vec{p} i}} \sum_{\left\{N_{j}\right\}, \sum_{j} j N_{j}=A} \theta\left(N_{i}-l\right) Q\left(N_{i}-l\right) \prod_{j \neq i}^{A} Q\left(N_{j}\right) .
$$

The step-function removes here those terms in $N_{i}$ for which $N_{i}<l$. So, the mean occupation number is 


$$
\left\langle\nu_{\vec{p} i}\right\rangle_{A}=\frac{1}{Z_{A}} \sum_{l=1}^{[A / i]}(\mp 1)^{l+1} e^{-\beta l \varepsilon_{\vec{p} i}} \sum_{\left\{N_{j \neq i}\right\}} \sum_{N_{i}=l}^{[A / i]} \delta\left(\sum_{j=1}^{A} j N_{j}-A\right) Q\left(N_{i}-l\right) \prod_{j \neq i}^{A} Q\left(N_{j}\right) .
$$

Changing the summation indices : $N_{i} \rightarrow N_{i}+l$, and noting that $N_{j}=0$ for all $j>A-i l$ as follows from the Kronecker symbol, we finally have

$$
\begin{aligned}
\left\langle\nu_{\vec{p} i}\right\rangle_{A} & =\frac{1}{Z_{A}} \sum_{l=1}^{[A / i]}(\mp 1)^{l+1} e^{-\beta l \varepsilon_{\vec{p} i}} \sum_{\left\{N_{j}\right\}, \sum_{j} j N_{j}=A-i l} \prod_{j=1}^{A-i l} Q\left(N_{j}\right) \\
& =\frac{1}{Z_{A}} \sum_{l=1}^{[A / i]}(\mp 1)^{l+1} e^{-\beta l \varepsilon_{\vec{p} i}} Z_{A-i l}
\end{aligned}
$$

where Eqs. (42), (77) were used to get the second equality in (83). After summing over momentum $\vec{p}$, the mean number of fragments of mass $i$ in the system of size $A$ can be related to the partition functions for systems of smaller size :

$$
\left\langle N_{i}\right\rangle_{A}=\frac{1}{Z_{A}} \sum_{l=1}^{[A / i]} f_{i l} Z_{A-i l}
$$

where the variables $f_{i l}$ were defined in Eq. (45). Note that 'il' denotes a single index corresponding to the product of 'i' by 'l'.

Using Eq. (84) the recurrence equations for the partition function $Z_{A}$ can be easily derived now from the conservation of the total nucleon number (36)

$$
Z_{A}=\frac{1}{A} \sum_{i=1}^{A} \sum_{l=1}^{[A / i]} i f_{i l} Z_{A-i l}
$$

where $Z_{0}=1$ according to (77). The partition function for an arbitrary $A$ is obtained by an iterative solution of the recurrence equations (85) starting from $Z_{0}=1$

$$
Z_{A}=\sum_{\left\{n_{i l}\right\}, \sum_{i, l} i n_{i l}=A} \prod_{i=1}^{A} \prod_{l=1}^{[A / i]} \frac{\left(f_{i l}\right)^{n_{i l}}}{l^{n_{i l}} n_{i l} !}
$$

The second moment of occupation numbers can be similarly derived from Eq. (79) with subsequent using of Eqs. (56) and (47)

$$
\begin{aligned}
\left\langle\nu_{\vec{p} i} \nu_{\vec{p}^{\prime} j}\right\rangle_{A} & =\frac{1}{Z_{A}} \sum_{k, l}(\mp 1)^{k+l} e^{-\beta k \varepsilon_{\vec{p} i}} e^{-\beta l \varepsilon_{\vec{p}^{\prime} j}} Z_{A-i k-j l} \\
& +\delta_{i j} \delta_{\vec{p} \vec{p}^{\prime}} \frac{1}{Z_{A}} \sum_{l}(\mp 1)^{l+1} e^{-\beta l \varepsilon_{\vec{p} i}} l Z_{A-i l} .
\end{aligned}
$$

Summing over all microscopic states of a fragment gives the second moment for the fragment number

$$
\left\langle N_{i} N_{j}\right\rangle_{A}=\delta_{i j} \frac{1}{Z_{A}} \sum_{l=1}^{[A / i]} l f_{i l} Z_{A-i l}+\frac{1}{Z_{A}} \sum_{k, l} f_{i k} f_{j l} Z_{A-i k-j l}
$$


where all functions $Z_{1}, Z_{2}, \ldots, Z_{A}$ are calculated from Eq. (85) with $f_{i l}$ defined for the system of $A$ nucleons. The knowledge of the first (84) and the second (88) multiplicity moments allows us to find the multiplicity dispersion

$$
\Delta\left(N_{i} N_{j}\right)_{A}=\left\langle N_{i} N_{j}\right\rangle_{A}-\left\langle N_{i}\right\rangle_{A}\left\langle N_{j}\right\rangle_{A}
$$

Similarly to (29), one can define the dispersion of occupation numbers in a given quantum state $\vec{p}$

$$
\left\langle\left(\Delta \nu_{\vec{p} i}\right)^{2}\right\rangle_{A}=\left\langle\left(\nu_{\vec{p} i}-\left\langle\nu_{\vec{p} i}\right\rangle_{A}\right)^{2}\right\rangle_{A}=\left\langle\nu_{\vec{p} \imath}^{2}\right\rangle_{A}-\left\langle\nu_{\vec{p} \imath}\right\rangle_{A}^{2}
$$

The mean squared term $\left\langle\nu_{\vec{p}}^{2}\right\rangle_{A}$ can be obtained for all statistics from Eq. (87), which reduces to

$$
\left\langle\nu_{\vec{p} i}^{2}\right\rangle_{A}=\frac{1}{Z_{A}} \sum_{j=1}^{[A / i]}(\mp 1)^{j+1} e^{-\beta j \varepsilon_{\vec{p} i}} Z_{A-i j}[\mp(j-1)+j]
$$

where the mean occupation numbers are defined by Eq. (83). So, the dispersion (90) for the case of Fermi gas fragments is

$$
\left\langle\left(\Delta \nu_{\vec{p} i}\right)^{2}\right\rangle_{A}=\left\langle\nu_{\vec{p} i}\right\rangle_{A}\left[1-\left\langle\nu_{\vec{p} i}\right\rangle_{A}\right]
$$

and coincides exactly with the result for the identical fermions (29). For the gas of Bose fragments, we have correspondingly (cp. (34)) :

$$
\left\langle\left(\Delta \nu_{\vec{p} i}\right)^{2}\right\rangle_{A}=\frac{1}{Z_{A}} \sum_{j=1}^{[A / i]} e^{-\beta j \varepsilon_{\vec{p} i}}(2 j-1) Z_{A-i j}-\left\langle\nu_{\vec{p} i}\right\rangle_{A}^{2}
$$

Note that the calculation of both the partition function $Z_{A}$ and ensemble averaging is carried out with the help of recurrence equations (85). However, these equations have still other representation related to the recurrence relations (16) for a system of $N$ identical bosons and fermions. By simple algebraic transformations, Eq. (85) can be reduced to the following form :

$$
Z_{A}=\frac{1}{A} \sum_{i=1}^{A} a_{i} Z_{A-i}
$$

where

$$
a_{i}=\sum_{k=1}^{i} \sum_{l=1}^{i} \delta(i-k l) k f_{k l}
$$

It is seen that Eq. (94) formally coincides with the recurrence relations (16), for which the multinomial sum (18) holds. Therefore, the partition function $Z_{A}$ of the canonical ensemble in the QSM of multifragmentation can be presented as the multinomial sum (18) with the redefined variables $a_{i}$ and an evident change $: N \rightarrow A$. The recurrence equations (94) can be considered as a set of $A$ linear equations with $A$ unknown values $Z_{1}, Z_{2}, \ldots, Z_{A}$ : 


$$
j Z_{j}-\sum_{i=1}^{j-1} a_{i} Z_{j-i}=a_{j}, \quad j=1,2, \ldots, A .
$$

Solving (96) by the Kramer method, we get the solution for the partition function (77) as a matrix determinant formed by $a_{i}$ coefficients (95)

$$
Z_{A}=\frac{1}{A !}\left|\begin{array}{lcccc}
a_{A} & -a_{1} & -a_{2} & \ldots & -a_{A-1} \\
a_{A-1} & A-1 & -a_{1} & \ldots & -a_{A-2} \\
a_{A-2} & 0 & A-2 & \ldots & -a_{A-3} \\
\vdots & \vdots & \vdots & \ddots & \vdots \\
a_{1} & 0 & 0 & \ldots & 1
\end{array}\right| .
$$

The partition function $Q_{N}$ for the system of $N$ Bose (Fermi) identical particles (16) can be also presented in the form of matrix determinant (97) with coefficients : $a_{i}=f_{i}$, and the evident change : $A \rightarrow N, Z_{A} \rightarrow Q_{N}$. Thus, the multinomial sum (18) can be unambiguously reduced to the matrix determinant (97).

The limiting case of the Boltzmann statistics can also be obtained in the way presented above. The explicit form of the canonical ensemble partition function for multifragmentation of the Boltzmann fragments is

$$
Z_{A}^{B}=\frac{1}{A !} \sum_{\left\{N_{j}\right\} \sum_{j} j N_{j}=A} \frac{A !}{\prod_{k=1}^{A} N_{k} !} \sum_{\left\{\nu_{\vec{p} i}\right\}} \prod_{k=1}^{A}\left(\frac{N_{k} !}{\prod_{\vec{p}} \nu_{\vec{p} k} !}\right) e^{-\beta E_{\left\{\nu_{\vec{p} i}\right\} N_{1}, N_{2}, \ldots, N_{A}}}
$$

where the total energy $E_{\left\{\nu_{\vec{p}}\right\} N_{1}, \ldots, N_{A}}$ is given by Eq. (38) and the occupation numbers take values $\nu_{\vec{p} i}=0,1, \ldots, N_{i}$. The ensemble averaging is also defined up to a permutation factor. Making use of the substitution (24) for Eqs. (85), (86), the partition function (98) results in the multinomial sum for which the following recurrence relations are fulfilled [20]

$$
Z_{A}^{B}=\sum_{\left\{N_{i}\right\}, \sum_{j} j N_{j}=A} \prod_{i=1}^{A} \frac{\omega_{i}^{N_{i}}}{N_{i} !}=\frac{1}{A} \sum_{i=1}^{A} i \omega_{i} Z_{A-i}^{B}
$$

$\omega_{i}=f_{i 1}$ in (99) denotes the partition function for ideal fragments of the $i$-th species (also the integer numbers $n_{i 1}$ were denoted as $N_{i}$ ).

The recurrence equations of this type were first applied to the multifragmentation process by Chase and Mekjian [18,19]. These equations were motivated by the Boltzmann treatment of fragments with phenomenologically postulated thermodynamic variable $\omega_{i}$ (the so-called tuning parameter). The partition function $Z_{A}^{B}$ in the form (97) remains unchanged but now : $a_{i}=i \omega_{i}$, for $i=1, \ldots, A$. The Boltzmann substitution (24) into expressions for the mean multiplicity of occupation numbers (83) and fragments numbers (84) gives rise to

$$
\begin{aligned}
\left\langle\nu_{\vec{p}}\right\rangle_{A}^{B} & =e^{-\beta \varepsilon_{\vec{p} i}} \frac{Z_{A-i}^{B}}{Z_{A}^{B}}, \quad i=1,2, \ldots, A \\
\left\langle N_{i}\right\rangle_{A}^{B} & =\omega_{i} \frac{Z_{A-i}^{B}}{Z_{A}^{B}} .
\end{aligned}
$$


For the second moments of the occupation numbers (87), their dispersion (90) and the fragment numbers (88), we have similarly

$$
\begin{aligned}
\left\langle\nu_{\vec{p} \imath} \nu_{\vec{p} j}\right\rangle_{A}^{B} & =e^{-\beta \varepsilon_{\vec{p} i}} e^{-\beta \varepsilon_{\vec{p}^{\prime} j}} \frac{Z_{A-i-j}^{B}}{Z_{A}^{B}}+\delta_{i j} \delta_{\vec{p} \vec{p}^{\prime}} e^{-\beta \varepsilon_{\vec{p} i}} \frac{Z_{A-i}^{B}}{Z_{A}^{B}} \\
\left\langle\left(\Delta \nu_{\vec{p} i}\right)^{2}\right\rangle_{A}^{B} & =\left\langle\nu_{\vec{p} i}\right\rangle_{A}\left[1-\left\langle\nu_{\vec{p} \imath}\right\rangle_{A}\right]+e^{-2 \beta \varepsilon_{\vec{p} i}} \frac{Z_{A-2 i}^{B}}{Z_{A}^{B}} \\
\left\langle N_{i} N_{j}\right\rangle_{A}^{B} & =\omega_{i} \omega_{j} \frac{Z_{A-i-j}^{B}}{Z_{A}^{B}}+\delta_{i j} \delta_{\vec{p} p^{\prime}} \quad \omega_{i} \frac{Z_{A-i}^{B}}{Z_{A}^{B}} .
\end{aligned}
$$

Let us now illustrate the influence of quantum statistics on various characteristics of nuclear fragmentation in terms of the QSM. Let us consider three versions of the model when all produced fragments are treated as either fermions, bosons or classical Boltzmann particles. Note that all fragments are assumed to be spinless, i.e., the spin degeneration factor is put equal one. In the results presented here, the Coulomb interaction potential is taken into account in the approximation (75) and experimental values of the binding energies $b_{i}$ are used everywhere unless opposite is said. Internal fragment excitation is neglected. Volume of the fragmenting nuclear system $V$ is regarded as a free parameter and its value is given in terms of the volume of a system at the normal nuclear density : $V / V_{0}=V \rho_{0} / A$.

The momentum dependence of the mean occupation numbers $\left\langle\nu_{\vec{p} i}\right\rangle$ is given in Fig. 1 for different fragments produced in the multifragmentation of system with $A=200$ nucleons. Bose statistics enhances noticeably the yield of light fragments with low momentum $(p<$ $150 \mathrm{MeV} / \mathrm{c}$ ) but this enhancement practically vanishes for IMF's. One should note that to get the observable momentum spectra for fragments, the given values of $\left\langle\nu_{\vec{p} \imath}\right\rangle$ should be multiplied by the phase factor $p^{2}$.

Temperature dependence of the mean fragment multiplicity $\left\langle N_{i}\right\rangle$ for the same system is presented in Fig. 2. Though the effect of quantum statistics slightly increases with temperature $T$, it is seen in fact only for nucleons. This is caused by the fact that one needs many identical particles to get a sizable quantum effect but the mean multiplicity for fragments is typically small as can be seen from Fig. 2. Note that the influence of quantum statistics on the mean fragment multiplicity is considerably less than that of uncertainty in the choice of the volume $V$ of the fragmenting system. The mean multiplicity of the lightest fragments grows gradually with increasing violence of the collision, while the IMF's exhibit clearly the "rise and fall" behavior observed experimentally [41]. The maximum in the $T$-dependence of $\left\langle N_{i}\right\rangle$ of IMF's is slightly shifted towards higher temperatures with increasing size of the fragment.

The "rise and fall" behavior noted above is manifested again in the $T$-dependence of the total mean IMF multiplicity defined as : $\langle M\rangle=\sum_{i=6}^{40}\left\langle N_{i}\right\rangle$ (see Fig. 3). All three model versions give practically the same results and demonstrate rather strong dependence of the maximum on the volume of the system. Due to large contribution of nucleons, the mean multiplicity of all fragments : $\langle m\rangle=\sum_{i=1}^{A}\left\langle N_{i}\right\rangle$, is an increasing function of $T$ with abrupt growth above the threshold of IMF production. This peculiar structure of $\langle m\rangle(T)$-curve is manifested stronger for heavier systems, as follows from the same Fig. 3.

Fluctuations in the region of phase transition behave in a special manner. To approach 
this issue, one can generalize the correlation characteristic $\eta(32$ ) to the case of fragment mixture

$$
\eta_{i}=\frac{\sum_{\vec{p}}\left[\left\langle\left(\Delta \nu_{\vec{p} i}\right)^{2}\right\rangle_{A}-\left\langle\nu_{\vec{p} i}\right\rangle_{A}\right]}{\sum_{\vec{p}}\left\langle\nu_{\vec{p} i}\right\rangle_{A}}
$$

Using (92), we obtain for the Fermi gas

$$
\eta_{i}=-\frac{1}{\left\langle N_{i}\right\rangle_{A}} \sum_{\vec{p}}\left\langle\nu_{\vec{p} i}\right\rangle_{A}^{2}
$$

i.e., $\eta_{i}$ is negative for all values of $T, V$ and $\eta_{A}=-1$ in the limit $T \rightarrow 0$. Using (93), one obtains for the Bose gas

$$
\eta_{i}=\frac{1}{\left\langle N_{i}\right\rangle_{A}}\left\{\frac{1}{Z_{A}} \sum_{l=1}^{[A / i]} f_{i l} 2(l-1) Z_{A-i l}-\sum_{\vec{p}}\left\langle\nu_{\vec{p}}\right\rangle_{A}^{2}\right\} .
$$

In the Boltzmann limit, this correlation characteristics is

$$
\eta_{i}^{B}=\frac{1}{\left\langle N_{i}\right\rangle_{A}}\left\{f_{i 2} \frac{Z_{A-2 i}^{B}}{Z_{A}^{B}}-\sum_{\vec{p}}\left\langle\nu_{\vec{p} \imath}\right\rangle_{A}^{2}\right\}
$$

and differs from the Fermi gas case (106) by a positive correction term.

In Fig. 4 the temperature dependence of $\eta_{i}$ is presented for various fragments. Indeed, there is a striking difference between the cases of Bose and Fermi statistics and this difference is maximal at the temperature corresponding to the maximal yield of this fragment $i$ going to zero. As noted above, $\eta<0$ for the Fermi gas. Beside the lightest fragment $i=1$, the Boltzmann limit follows very closely the Fermi case and the difference between them becomes negligible for heaviest IMF's. Because the nucleon component is dominant and $\left\langle N_{1}\right\rangle$ is rather large, its $\eta$-behavior looks like that for $\left\langle N_{1}\right\rangle$ identical particles with correlations which are positive, negative or zero for bosons, fermions or Boltzmann particles, respectively. Deviation of $\eta_{1}$ from zero value for the classical case is due to the admixture of other fragments.

Let us now consider more general characteristics of correlations by summing $\eta_{i}$ over a certain interval of $i$. In particular, after summing over all $m$ fragments in the numerator and denominator of (105) we have

$$
\eta(m)=\frac{\sum_{i=1}^{A} \sum_{\vec{p}}\left\langle\left(\Delta \nu_{\vec{p} i}\right)^{2}\right\rangle_{A}-\langle m\rangle_{A}}{\langle m\rangle_{A}}
$$

Analogously, the summation over IMF's yields

$$
\eta(M)=\frac{\sum_{i=\mu_{1}}^{\mu_{2}} \sum_{\vec{p}}\left\langle\left(\Delta \nu_{\vec{p} i}\right)^{2}\right\rangle_{A}-\langle M\rangle_{A}}{\langle M\rangle_{A}}
$$


where $\mu_{1}=6$ and $\mu_{2}=40$ were chosen.

As follows from the results presented in Fig. 5, the above mentioned properties of the occupation number distributions survive in more global characteristics $\eta(M)$ and $\eta(m)$. Since the contribution of nucleons is dominant, therefore $\eta(m) \approx \eta_{1}$ for temperatures higher than about $2-3 \mathrm{MeV}$. Note that $\eta(\mathrm{m})$ goes down when $T \rightarrow 0$. Under this condition, the system of $A$ identical particles exists as a unique fragment and $\eta_{A}$ goes to its limiting value -1 for all three statistics as discussed above. In other words, in the limit $T \rightarrow 0$ it behaves like a closed-packed system [28]. It is natural that all correlation effects caused by quantum statistics are seen more clearly in the denser systems corresponding to smaller values of $V$.

Rather similar correlation characteristics can be defined for the multiplicity distribution of a fragment $i$

$$
\gamma_{i}=\Delta\left(N_{i}^{2}\right)_{A}-\left\langle N_{i}\right\rangle_{A}
$$

where the dispersion $\Delta\left(N_{i}^{2}\right)_{A}$ is calculated according to Eq. (89). Note that as compared to the definition of $\eta_{i}$, the factor $\left\langle N_{i}\right\rangle$ is dropped and the quantity $\gamma_{i}$, which is the "shifted" multiplicity dispersion, may be considered as a shape distribution characteristics.

The calculated $\gamma_{i}$ are displayed in Fig. 6. For the case of Poisson distribution, the dispersion (89) equals the mean multiplicity (84) and therefore $\gamma_{i}=0$. Such a situation is realised for a dominating nucleon component in the Boltzmann gas mixture where $\gamma_{1} \approx$ 0 . Visible differences appear for quantum cases. However, because in the whole mixture of different (non-identical) fragments the fraction of specific identical fragments is small, therefore all three statistics give similar results. As can be seen from Fig. 6 for $i \neq 1$ and temperature just above the threshold, $\gamma_{i}(T)$ is positive and has a spike-like structure showing that the fragment mass distribution is getting much wider at the temperature around the phase transition. At higher temperature, $\gamma_{i}(T)$ is negative, i.e., the distribution is subPoissonian [22] and approaches asymptotically the Poisson distribution.

By suming over $i$ one can define a more global characteristics for IMF's

$$
\gamma(M)=\sum_{i=\mu_{1}}^{\mu_{2}} \Delta\left(N_{i}^{2}\right)_{A}-\langle M\rangle_{A}
$$

and for all produced fragments

$$
\gamma(m)=\sum_{i=1}^{A} \Delta\left(N_{i}^{2}\right)_{A}-\langle m\rangle_{A} .
$$

As is seen in Fig. 7, the above mentioned features are found in the $\gamma(M)$ and $\gamma(m)$ reduced dispersions. The IMF distributions near the transition temperature have an enlarged multiplicity width and this width increases with increasing the freeze-out density of the fragmenting system.

\section{B. Thermodynamic quantities}

Let us now express the global thermodynamic averages characterizing the equation of state of nuclear matter through the partition functions $Z_{A}$. The average energy of the system is 


$$
\left.E=\left.T^{2} \frac{\partial \ln Z_{A}}{\partial T}\right|_{V}=\left\langle E_{\left\{\nu_{\vec{p}}\right\}}\right\} N_{1} \ldots, N_{A}\right\rangle_{A}
$$

After the substitution of the average energy (38) into expression for the ensemble averaging (79), we have

$$
E=\sum_{i, \vec{p}} \varepsilon_{\vec{p} \imath}\left\langle\nu_{\vec{p} i}\right\rangle_{A}=\frac{1}{Z_{A}} \sum_{i=1}^{A} \sum_{l=1}^{[A / i]} \overline{\varepsilon_{i l}} Z_{A-i l}
$$

Here Eq. (83) was used in getting the second equality and the function $\overline{\varepsilon_{i l}}$ was calculated according to Eq. (55). Making use of Eq. (84), the average energy in the system (115) can be related to the multiplicity of fragments and to their average energies

$$
\begin{aligned}
& E=\sum_{i=1}^{A} \overline{\varepsilon_{i}}\left\langle N_{i}\right\rangle_{A} \\
& \overline{\varepsilon_{i}}=\frac{1}{\overline{f_{i}}} \sum_{l=1}^{[A / i]} \overline{\varepsilon_{i l}} Z_{A-i l} \\
& \overline{f_{i}}=\sum_{j=1}^{[A / i]} f_{i j} Z_{A-i j} .
\end{aligned}
$$

The evaluation of functions $\overline{\varepsilon_{i l}}$ and $f_{i l}$ is considered in the Appendix B.

The pressure in the multifragmenting system

$$
P=\left.T \frac{\partial \ln Z_{A}}{\partial V}\right|_{T}=-\left\langle\frac{\partial E_{\left\{\nu_{\vec{p}}\right\}} N_{1} \ldots, N_{A}}{\partial V}\right\rangle_{A}=-\sum_{i, \vec{p}} \frac{\partial \varepsilon_{\vec{p} i}}{\partial V}\left\langle\nu_{\vec{p} i}\right\rangle_{A}
$$

can be related to quantities averaged over single-particle momentum, if Eq. (83) is used for the mean occupation numbers

$$
\begin{aligned}
P & =\frac{1}{Z_{A}} \sum_{i=1}^{A} \sum_{l=1}^{[A / i]} \overline{P_{i l}} Z_{A-i l}=\sum_{i=1}^{A} \overline{P_{i}}\left\langle N_{i}\right\rangle_{A} \\
\overline{P_{i}} & =\frac{1}{\overline{f_{i}}} \sum_{l=1}^{[A / i]} \overline{P_{i l}} Z_{A-i l}
\end{aligned}
$$

with the function $\overline{P_{i l}}$ calculated using Eq. (59).

The heat capacity at the constant volume can be found in the similar way

$$
\begin{aligned}
C_{V}=\left.\frac{\partial E}{\partial T}\right|_{V} & =\frac{\left.\left\langle E_{\left\{N_{\vec{p} i}\right\}}^{2}\right\} N_{1}, \ldots, N_{A}\right\rangle_{A}-\left\langle E_{\left.\left\{N_{\vec{p} \imath}\right\} N_{1}, \ldots, N_{A}\right\rangle_{A}^{2}}\right.}{T^{2}} \\
& =\beta^{2} \sum_{i, j, \vec{p}, \vec{p}^{\prime}} \Delta\left(\nu_{\vec{p} \imath} \nu_{\vec{p}^{\prime} j}\right)_{A} \varepsilon_{\vec{p} i} \varepsilon_{\vec{p}^{\prime} j} .
\end{aligned}
$$

Averaging (122) over momentum and using Eqs. (89), (83) and (87), we finally obtain 


$$
C_{V}=\beta^{2}\left[-E^{2}+\frac{1}{Z_{A}} \sum_{i, j} \sum_{k, l} \overline{\varepsilon_{i k}} \overline{\varepsilon_{j l}} Z_{A-i k-j l}+\frac{1}{Z_{A}} \sum_{i, l} l \overline{\varepsilon_{i l}^{2}} Z_{A-i l}\right]
$$

where $\overline{\varepsilon_{i l}}$ and $\overline{\varepsilon_{i l}^{2}}$ are defined by Eqs. (55) and (63), respectively.

The classical limit of the derived thermodynamics relations can be found according to the procedure described above. The average energy in the QSM for the Boltzmann fragments is obtained from Eqs. (116), (117) after the Boltzmann substitution (24)

$$
E^{B}=\sum_{i=1}^{A} \overline{\varepsilon_{i}}\left\langle N_{i}\right\rangle_{A}^{B}
$$

where $\overline{\varepsilon_{i}}$ is defined by (69). The pressure is given by Eqs. (120), (121) :

$$
P^{B}=\sum_{i=1}^{A} \overline{P_{i}}\left\langle N_{i}\right\rangle_{A}^{B}
$$

and $\overline{P_{i}}$ is defined by (71). After applying the same procedure and using Eqs. (89), (101), (104), (124), the heat capacity (123) reduces to

$$
C_{V}^{B}=\beta^{2} \sum_{i j} \Delta^{B}\left(N_{i} N_{j}\right)_{A} \overline{\varepsilon_{i}} \overline{\varepsilon_{j}}+\beta^{2} \sum_{i=1}^{A}\left(\overline{\varepsilon_{i}^{2}}-{\overline{\varepsilon_{i}}}^{2}\right)\left\langle N_{i}\right\rangle_{A}^{B}
$$

where $\overline{\varepsilon_{i}^{2}}$ is given by Eq. (73).

An example of thermodynamic characteristics calculated within the QSM of multifragmentation is presented in Fig. 8. There is a narrow region of temperatures where the average energy per nucleon in the system, $E / A$, is growing very quickly. This region clearly corresponds to a sharp maximum of the heat capacity $C_{V}$ at a constant volume indicating the first order phase transition. The finite width of $C_{V}$ reflects the finite size of the system under consideration. The position of the maximum of $C_{V}$ depends noticeably on the fragmenting volume and is correlated with the position of maximum in IMF multiplicity (see Fig. 2). One should note that the quick increase in $E / A$ as a function of $T$ is unambiguously related to the "plateau" region of the inverse function $T(E / A)$ which is called sometimes the caloric curve 1 [42]. As follows from Fig. 8, the plateau region is more distinct for heavy systems.

The volume dependence of the pressure is shown in Fig. 9. The presented family of isotherms clearly indicates the first order phase transition in the fragmenting system. Such a behavior in the QSM is caused solely by the Coulomb interaction of fragments. If the Coulomb potential (75) is neglected, then the isotherms follow the case of classical ideal gas (see r.h.s. in Fig. 9). One should stress also that the considered characteristics of the equation of state are insensitive to quantum statistics effects.

\footnotetext{
${ }^{1}$ To be exact, the caloric curve is the $T\left(E^{*} / A\right)$ dependence but the excitation energy per nucleon $E^{*} / A$ differs from $E / A$ by a constant value which is equal to the binding energy of the fragmenting nucleus.
} 


\section{IMF multiplicity distribution}

In the canonical ensemble, a nuclear system of $A$ nucleons may have $A$ different species of fragments : $1 \leq i \leq A$. A number of intermediate mass fragments $M$ involving : $\mu_{1} \leq i \leq \mu_{2}$, is defined as

$$
M=\sum_{i \in \mu} N_{i}
$$

where $i$ is taken from the IMF range $\mu=\left(\mu_{1}, \ldots, \mu_{2}\right)$. So, a number of IMF's for the system of $A$ nucleons may take the following values

$$
M=0,1, \ldots,\left[A / \mu_{1}\right] .
$$

The probability to find exactly $M$ fragments in the mass range $\mu$ is

$$
P_{A}(M)=\frac{Z_{A}(M)}{Z_{A}}
$$

where the partition function $Z_{A}$ is given by Eq. (77) and the new partition function $Z_{A}(M)$, characterizing the probability of a system to be in a state with $M$, is defined as follows

$$
Z_{A}(M)=\sum_{\left\{N_{j}\right\}, \sum_{j} j N_{j}=A} \delta\left(\sum_{i \in \mu} N_{i}-M\right) \prod_{l=1}^{A} Q\left(N_{l}\right) .
$$

Let us derive the recurrence relations that are needed for calculating this new partition function $Z_{A}(M)$.

By the direct account of the restriction (127), let us generalize the mean occupation numbers of the $A$ nucleon system to the case of $M$ IMF's

$$
\left\langle\nu_{\vec{p} \imath}\right\rangle_{A}(M)=\frac{1}{Z_{A}(M)} \sum_{\left\{N_{j}\right\} \sum_{j} j N_{j}=A} \delta\left(\sum_{i \in \mu} N_{i}-M\right)\left\langle\nu_{\vec{p} \imath}\right\rangle_{N_{1}, \ldots, N_{A}} \prod_{l=1}^{A} Q\left(N_{l}\right)
$$

where $Q\left(N_{l}\right)$ is related to the variable $x_{\vec{p} i}$ by Eq. (9). By differentiating the logarithm of the partition function (130) with respect to $x_{\vec{p} \imath}$ (see the definition (80), we get for the mean occupation numbers (131):

$$
\left\langle\nu_{\vec{p} i}\right\rangle_{A}(M)=x_{\vec{p} i} \frac{\partial \ln Z_{A}(M)}{\partial x_{\vec{p} i}} .
$$

The product of the partition functions $Q\left(N_{i}\right)$ for subsystems of identical fragments (43) enters the definition of the partition function (130). Each term of this product satisfies the recurrence relation (10), so we have

$$
x_{\vec{p} i} \frac{\partial Q\left(N_{i}\right)}{\partial x_{\vec{p} i}}=\sum_{l=1}^{N_{i}}(\mp 1)^{l+1} x_{\vec{p} i}^{l} Q\left(N_{i}-l\right), \quad i=1,2, \ldots, A .
$$


The direct differentiation of the partition function (130) with respect to $x_{\vec{p} i}$ gives for all allowed values of $M$

$$
x_{\vec{p} i} \frac{\partial Z_{A}(M)}{\partial x_{\vec{p} i}}=\sum_{\left\{N_{j}\right\}, \sum_{j} j N_{j}=A} \delta\left(\sum_{k \in \mu} N_{k}-M\right)\left(x_{\vec{p} i} \frac{\partial \ln Q\left(N_{i}\right)}{\partial x_{\vec{p} i}}\right) \prod_{l=1}^{A} Q\left(N_{l}\right) .
$$

Substituting (133) into (134) and using the method employed above (see Eqs. (81)-(83)) to calculate $\left\langle\nu_{\vec{p} i}\right\rangle$, one finds for the case $M=0$

$$
\begin{array}{ll}
x_{\vec{p} i} \frac{\partial Z_{A}(M=0)}{\partial x_{\vec{p} i}}=\sum_{l=1}^{[A / i]}(\mp 1)^{l+1} x_{\vec{p} i}^{l} Z_{A-i l}(M=0), & i \notin \mu \\
x_{\vec{p} i} \frac{\partial Z_{A}(M=0)}{\partial x_{\vec{p} i}}=0, & i \in \mu .
\end{array}
$$

By differentiating (134), we obtain in the case of $M \neq 0$ and $i \in \mu$

$$
x_{\vec{p} i} \frac{\partial Z_{A}(M)}{\partial x_{\vec{p} i}}=\sum_{l=1}^{\min ([A / i], M)}(\mp 1)^{l+1} x_{\vec{p} i}^{l} Z_{A-i l}(M-l) .
$$

The mean number of fragments $i$ for the $A$ nucleon system with the fixed number $M$ of IMF's is

$$
\left\langle N_{i}\right\rangle_{A}(M)=\sum_{\vec{p}}\left\langle\nu_{\vec{p} i}\right\rangle_{A}(M)
$$

where the mean occupation numbers are determined by (131), (132). The mean fragment number (138) satisfies the restrictions (36) and (127). By the substitution of Eqs. (135), (136) into (132) and then into Eq. (138) we have for the mean fragment multiplicity in the case of $M=0$

$$
\begin{array}{ll}
\left\langle N_{i}\right\rangle_{A}(M=0)=\frac{1}{Z_{A}(M=0)} \sum_{l=1}^{[A / i]} f_{i l} Z_{A-i l}(M=0), & i \notin \mu \\
\left\langle N_{i}\right\rangle_{A}(M=0)=0, & i \in \mu .
\end{array}
$$

Here $f_{i l}$ is calculated according to (45).

To find the average fragment multiplicity in the case of $M \neq 0$ and $i \in \mu$, one inserts Eq. (137) into (132) what after using (138) results in the following relation :

$$
\left\langle N_{i}\right\rangle_{A}(M)=\frac{1}{Z_{A}(M)} \sum_{l=1}^{\min ([A / i], M)} f_{i l} Z_{A-i l}(M-l) .
$$

In accordance with (130), we have the following relations. For systems with the nucleon number $j<\mu_{1}$ and the IMF number $M=0$

$$
Z_{j}(M=0)=Z_{j}, \quad j=0,1, \ldots, \mu_{1}-1 .
$$

For system with $j<\mu_{1} \cdot M$ and $M \neq 0$ one has 


$$
Z_{j}(M)=0, \quad j=0,1, \ldots, \mu_{1} \cdot M-1 .
$$

Finally, for the "conditional" partition function $Z_{j}(M)$ in the case of $M=0$, after the substitution of the mean fragment numbers (139), (141) in the the conservation law of the total number of nucleons (36), we have the following recurrence equations

$$
Z_{j}(M=0)=\frac{1}{j} \sum_{i \notin \mu} \sum_{l=1}^{[j / i]} i f_{i l} Z_{j-i l}(M=0), \quad j=\mu_{1}, \ldots, A
$$

The recurrence equations for the case of any $M \neq 0,\left(M=1,2, \ldots,\left[j / \mu_{1}\right]\right)$ are obtained similarly by making use of the restriction (127) and Eq. (141) :

$$
Z_{j}(M)=\frac{1}{M} \sum_{i \in \mu} \sum_{l=1}^{\min ([j / i], M)} f_{i l} Z_{j-i l}(M-l), \quad j=\mu_{1} \cdot M, \ldots, A
$$

If one now divides Eqs. (142) and (145) by the partition function $Z_{j}$ and applies the probability definition (129), we get the recurrence relations for probability to find exactly $M$ fragments among IMF's. If $M=0$

$$
\begin{array}{ll}
P_{j}(M=0)=1, & j=0,1, \ldots, \mu_{1}-1 \\
P_{j}(M=0)=\frac{1}{j} \sum_{i \notin \mu} \sum_{l=1}^{[j / i]} P_{j-i l}(M=0) i f_{i l} \frac{Z_{j-i l}}{Z_{j}}, & j=\mu_{1}, \ldots, A .
\end{array}
$$

If $M \neq 0$ then

$$
\begin{array}{ll}
P_{j}(M)=0, & j=0,1, \ldots, \mu_{1} \cdot M-1 \\
P_{j}(M)=\frac{1}{M} \sum_{i \in \mu} \sum_{l=1}^{\min ([j / i], M)} P_{j-i l}(M-l) f_{i l} \frac{Z_{j-i l}}{Z_{j}}, & j=\mu_{1} \cdot M, \ldots, A .
\end{array}
$$

In the Boltzmann limit, the IMF multiplicity distributions and related equations can be easily derived from the above derived quantum results by means of the substitution (24). In particular, the equalities (142), (143) for the partition function are not changed and Eqs. (144) and (145) reduce to the following equations for $M=0$ and $M \neq 0$, respectively :

$$
\begin{array}{rlrl}
Z_{j}^{B}(M=0) & =\frac{1}{j} \sum_{k \notin \mu} k \omega_{k} Z_{j-k}^{B}(M=0), & & j=\mu_{1}, \ldots, A \\
Z_{j}^{B}(M)=\frac{1}{M} \sum_{k \in \mu} \omega_{k} Z_{j-k}^{B}(M-1), & j & =\mu_{1} \cdot M, \ldots, A .
\end{array}
$$

Similarly, the recurrence equations for the probability to find $M$ IMF's in the classical model of multifragmentation result from Eqs. (146)-(149). If $M=0$

$$
\begin{aligned}
P_{j}(M=0)=1, & j=0,1, \ldots, \mu_{1}-1 \\
P_{j}(M=0)=\frac{1}{j} \sum_{k \notin \mu} P_{j-k}(M=0) k \omega_{k} \frac{Z_{j-k}}{Z_{j}}, & j=\mu_{1}, \ldots, A .
\end{aligned}
$$


If $M \neq 0$

$$
\begin{aligned}
P_{j}(M) & =0, & j & =0,1, \ldots, \mu_{1} \cdot M-1 \\
P_{j}(M) & =\frac{1}{M} \sum_{k \in \mu} P_{j-k}(M-1) \omega_{k} \frac{Z_{j-k}}{Z_{j}}, & j & =\mu_{1} \cdot M, \ldots, A .
\end{aligned}
$$

One should note that the multiplicity distributions of IMF's within the Boltzmann statistics have been recently studied in Refs. [43, 44]. Their equation coincides with our Eq. (155) but Eqs. (152), (153) for $M=0$ have not been found and the normalization condition $\sum_{M} P(M)=1$ were used to get the full distribution in 43,44.

The multiplicity distributions of IMF's $\left(\mu_{1}=6, \mu_{2}=40\right)$ calculated within the QSM of multifragmentation are presented in Fig. 10. It is of great interest that the $M$-distribution sharply changes its shape in the vicinity of the phase transition temperature defined by the maximum of the heat capacity. These results are in full agreement with the discussion of the reduced dispersions in Figs. 6, 7 and reveal the different IMF distributions in different phases as it would be expected for the phase transition. Details of the multiplicity distributions depend on the mass number $A$ and the size of the system, being weakly sensitive to quantum statistics of fragments. Some local irregularities and the appearance of two maxima in the transition curve of IMF multiplicity distributions are caused by the use of experimental values for the fragment binding energy. If the Coulomb fragment interaction is neglected and a smoothed approximation is used for the binding energies, the QSM with the Boltzmann statistics reproduces exactly the numerical results in [43,44].

\section{Total multiplicity distribution}

The total fragment multiplicity $m$ associated with the multifragmentation of a system of the size $A$ is defined similarly to (127)

$$
m=\sum_{i \in \mu} N_{i}
$$

except that the region $\mu$ is now :

$$
\mu=(1,2, \ldots, A)
$$

i.e., the events with $m=0$ are absent due to the nucleon number conservation. The probability to find $m$ fragments is defined by the same Eq. (129) with the conditional probability (130). From this follows immediately that in the case of $m=0$ the only non-zero partition function is that for $j=0$ :

$$
Z_{j}(m)=\delta_{j, 0}, \quad j=0,1, \ldots, A .
$$

For the partition functions with $j<m$, the following relations are valid

$$
Z_{j}(m)=0, \quad m=1,2, \ldots, A, \quad j=0,1, \ldots, m-1 .
$$

In this case, the recurrence equations (133) and the derivative of the partition function (134) still hold but the region $\mu$ is defined by Eq. (157). Similar transformation of Eq. (134) with using (133) gives for the particular value $m=1$ 


$$
\begin{array}{ll}
x_{\vec{p} i} \frac{\partial Z_{A}(m)}{\partial x_{\vec{p} i}}=0, & i=1,2, \ldots, A-1 \\
x_{\vec{p} i} \frac{\partial Z_{A}(m)}{\partial x_{\vec{p} i}}=x_{\vec{p} i} Z_{A-i}(m-1), & i=A .
\end{array}
$$

For derivatives (134) with $m=2,3, \ldots, A$ we have

$$
\begin{array}{rlrl}
x_{\vec{p} \imath} \frac{\partial Z_{A}(m)}{\partial x_{\vec{p} i}}=\sum_{l=1}^{\min ([A / i], m)}(\mp 1)^{l+1} x_{\vec{p} i}^{l} Z_{A-i l}(m-l) & , & i=1,2, \ldots, A-m+1 \\
x_{\vec{p} \imath} \frac{\partial Z_{A}(m)}{\partial x_{\vec{p} i}}=0, & i=A-m+2, \ldots, A .
\end{array}
$$

According to the definition, the mean fragment number of the $A$ nucleon system with the total multiplicity $m$, for which two restrictions (36) and (156) are fulfilled, is

$$
\left\langle N_{i}\right\rangle_{A}(m)=\sum_{\vec{p}}\left\langle N_{\vec{p}}\right\rangle_{A}(m)
$$

By substituting (160) and (161) into Eq. (132), we get for the mean fragment number (164) in the case of $m=1$ :

$$
\begin{aligned}
\left\langle N_{i}\right\rangle_{A}(m) & =0, & i & =1,2, \ldots, A-1 \\
\left\langle N_{i}\right\rangle_{A}(m) & =f_{i 1} \frac{Z_{A-i}(m-1)}{Z_{A}(m)}, & i & =A
\end{aligned}
$$

with $f_{i 1}$ calculated according to (45). This quantity for the case of $m=2,3, \ldots, A$ is obtained by combining (162), (163) and (132)

$$
\begin{array}{rlrl}
\left\langle N_{i}\right\rangle_{A}(m)=\frac{1}{Z_{A}(m)} \sum_{l=1}^{\min ([A / i], m)} f_{i l} Z_{A-i l}(m-l), & & i=1,2, \ldots, A-m+1 \\
\left\langle N_{i}\right\rangle_{A}(m) & =0, & & i=A-m+2, \ldots, A
\end{array}
$$

with $f_{i l}$ defined again by (45).

As usually, the recurrence relations are derived from the restriction (36) to the $j$ nucleon system. From Eqs. (165), (166) we have for $m=1$

$$
Z_{j}(m)=f_{j 1}, \quad j=1, \ldots, A
$$

and similarly for $m=2,3, \ldots, A$, by using Eqs. (167) and (168), we have

$$
Z_{j}(m)=\frac{1}{j} \sum_{i=1}^{j-m+1} \sum_{l=1}^{\min ([j / i], m)} i f_{i l} Z_{j-i l}(m-l), \quad j=m, \ldots, A .
$$

Eqs. (158), (159) and (169), (170) form a complete set which is sufficient for calculating the partition function $Z_{A}(m)$ and therefore the multiplicity distributions $P(m)$.

The limiting case of the Boltzmann statistics for the total multiplicity distributions can be obtained from the appropriate quantum equations by means of the substitution (24). 
For the system of classical fragments, Eqs. (158), (159), (169) are not changed and Eq. (24) results in the following recurrence relations in the case of $m=2,3, \ldots, A$ :

$$
Z_{j}^{B}(m)=\frac{1}{j} \sum_{i=1}^{j-m+1} i \omega_{i} Z_{j-i}^{B}(m-1), \quad j=m, \ldots, A .
$$

The total multiplicity distributions of fragments within the QSM are exemplified in Fig. 11. General behavior of $m$-distributions is close to those noted before while discussing the IMF distributions (cp. to Fig. 10), however all curves became more smooth. The reason of this behavior is a dominance of individual nucleons what results also in somewhat stronger influence of the quantum statistics on the multiplicity distributions.

\section{CONCLUSIONS}

The model proposed in this work realizes for the first time the quantum statistical approach to the multifragmentation of excited finite nuclei in the framework of the canonical ensemble method. The mathematical basis of the model is the recurrence equation technique which allows, within the usually accepted physics assumptions, to solve a cumbersome problem of fragment partitioning exactly without involving complicated and time-consuming Monte Carlo methods. The QSM opens a possibility for calculating in the same framework various microscopic characteristics of occupation numbers, global thermodynamic variables specifying the equation of state, and different observables allowing for a comparison with the experiment. The exactly solvable technique makes such calculations transparent to the physics assumptions used. The QSM includes the limiting case of the classical Boltzmann statistics and in this case the QSM reduces to a class of solvable models developed earlier [18, 19, 31, 32].

In principle, effects of quantum statistics in the QSM are seen on the microscopic level in quantities like $\left\langle\nu_{i}\right\rangle,\left\langle\nu_{i}^{2}\right\rangle$, especially at temperatures corresponding to the maximal fragment yield. The strength of these effects is proportional to a number of available identical clusters of a given size. Thus, the effect is strongest for nucleons and is noticeably weaker for the IMF's. It is of interest that large uncertainties in the choice of the freeze-out volume do not kill these quantum statistical effects in the correlation characteristics $\eta_{i}$.

Global thermodynamic variables, which are insensitive to quantum statistics, exhibit nevertheless a rather sharp phase transition of the first order in the temperature region $\approx 6-$ $8 \mathrm{MeV}$ depending on the size of the system and on the value of the freeze-out volume $V / V_{0}$. In the QSM, the observed Van der Waals behavior is caused by the competition between attractive forces, included in the fragment binding energies, and the Coulomb interaction energy. It is noteworthy that at not so high temperatures, the value of $V / V_{0}=3$, being the standard value for the SMM calculations of the primary fragment partition [6], turns out to be in the thermodynamically unstable region : $\partial P / \partial V<0$. This implies that before getting an equilibrium solution in the model analysis, the Maxwell construction should be applied to the isotherms in this unstable region. The use of these Maxwellian isotherms is important for extracting true values of the freeze-out temperature of a system as well as for detailed studies of the caloric curve.

Fragment multiplicity is a very interesting observable, even though the quantum statistical effects are practically absent in the averaged quantities. The mean number $\left\langle N_{i}\right\rangle$ of 
IMF's clearly demonstrates the "rise and fall" behavior as a function of temperature. Fragment multiplicity dispersion has a maximum near the transition temperature corresponding to the maximum of heat capacity $C_{V}$ as a sign of the phase transition in finite systems to be dependent on the system size, freeze-out volume and fragment binding energies. These features are remarkably well seen in the change of the shape of the IMF multiplicity distribution in the transition region. The measurement of the HBT effect for IMF's which would be crucial for determining the size of a fragmenting system, seems to be a hard task due to a comparatively small number of identical particles. However, the model studies in this paper show where this effect is expected to be maximal. In addition, when searching for the HBT effect a more detailed quantity, the correlated particle spectrum $\left\langle N_{i}(\vec{p}) N_{i}(\vec{p})\right\rangle$ rather than the mean square multiplicity $\left\langle N_{i}^{2}\right\rangle$ should be investigated [22]. In any case, this issue deserves a special discussion. Making use of protons instead of the IMF's unfortunately does not help much because in a real experiment this component may be strongly enriched by non-equilibrium particles.

As a final remark, the model developed in this paper takes into account the mass number conservation while the conservation of the charge number may be quite important for the yield of specific isotopes or even for the equation of state. In particular, it was shown that the liquid-gas transition in the asymmetric nuclear matter is of the second order rather than of the first order as one would expect for neutron-proton symmetric matter [45]. This process is of particular interest because experimental data for $A u+C$ at $1 \mathrm{GeV}$ per nucleon [46] shows a much smoother phase transition than the first observation in the $A u+A u$ collision at $600 \mathrm{MeV}$ [41]. The recurrence equation technique allows one to consider simultaneously both mass and charge number conservation. Such a generalization has been done in Refs. [47,48 but only for the mean multiplicity of individual fragments within the Boltzmann statistics. Similar extension for calculating the multiplicity distributions of the identified IMF's would open a new way to study critical exponents, intermittence phenomenon and so on. This work is in progress now.

Acknowledgements : We are thankful to K.K. Gudima and A.A. Shanenko for useful discussions. The work was supported by the CNRS-JINR agreement No 96-28. 


\section{APPENDIX A: A USEFUL RECURRENCE RELATION}

As an auxiliary step, let us define the partition function $Q_{N}^{n_{\vec{p}}}$ for a system of $N$ Bose (Fermi) identical particles with the fixed number $n_{\vec{p}}$ of particles in a given single particle state $\vec{p}$

$$
Q_{N}^{n_{\vec{p}}}=\sum_{\left\{\nu_{\vec{p}}\right\}} \delta\left(\sum_{\vec{p}^{\prime}} \nu_{\vec{p}^{\prime}}-N\right) \delta\left(n_{\vec{p}}-\nu_{\vec{p}}\right) \prod_{\vec{p}^{\prime}} x_{\vec{p}^{\prime}}^{\nu_{\vec{p}^{\prime}}}
$$

where the conservation laws are accounted for by the Kronecker symbol and the allowed occupation numbers $\nu_{\vec{p}}$ are given by (5). Then, the total partition function (9) for $N$ identical particles can be expressed via auxiliary one (A1) as follows

$$
Q_{N}=\sum_{n_{\vec{p}}=0}^{\{1\},\{N\}} Q_{N}^{n_{\vec{p}}}
$$

where the upper limit in the sum is equal to $N$ or 1 for bosons or fermions, respectively. The sum in (A1) with respect to $\nu_{\vec{p}}$ is taken by means of delta functions

$$
\begin{aligned}
Q_{N}^{n_{\vec{p}}} & =\sum_{\left\{\nu_{\vec{p}^{\prime}}\right\}_{\vec{p}^{\prime} \neq \vec{p}}} \delta\left(\sum_{\vec{p}^{\prime} \neq \vec{p}} \nu_{\vec{p}^{\prime}}+n_{\vec{p}}-N\right) x_{\vec{p}}^{n_{\vec{p}}} \prod_{\vec{p}^{\prime} \neq \vec{p}} x_{\vec{p}^{\prime}}^{\nu_{\vec{p}^{\prime}}} \\
& =x_{\vec{p}}^{n_{\vec{p}}} \sum_{\left\{\nu_{\vec{p}^{\prime}}\right\}} \delta\left(\sum_{\vec{p}^{\prime}} \nu_{\vec{p}^{\prime}}-\left(N-n_{\vec{p}}\right)\right) \delta\left(\nu_{\vec{p}}\right) \prod_{\vec{p}^{\prime}} x_{\vec{p}^{\prime}}^{\nu_{\vec{p}^{\prime}}} .
\end{aligned}
$$

Comparing the r.h.s of (A3) to the definition (A1), one can see that here one can select out the partition function for $\left(N-n_{\vec{p}}\right)$ particles in the state $\vec{p}=0$

$$
Q_{N}^{n_{\vec{p}}}=x_{\vec{p}}^{n_{\vec{p}}} Q_{N-n_{\vec{p}}}^{n_{\vec{p}}=0}
$$

This will result in the recurrence relations for the partition function (A1) which are valid for both Bose and Fermi particles. Let us start with the boson case. After the substitution of (A4) into (A2), we have for the total partition function

$$
Q_{N}=Q_{N}^{n_{\vec{p}}=0}+\sum_{n_{\vec{p}}=1}^{N} x_{\vec{p}}^{n_{\vec{p}}} Q_{N-n_{\vec{p}}}^{n_{\vec{p}}=0}
$$

Changing the summation indices in the second term on the r.h.s. of (A5) and using the recurrence relations (A4), we obtain

$$
Q_{N}=Q_{N}^{n_{\vec{p}}=0}+x_{\vec{p}} \sum_{n_{\vec{p}}=0}^{N-1} Q_{N-1}^{n_{\vec{p}}}=Q_{N}^{n_{\vec{p}}=0}+x_{\vec{p}} Q_{N-1}
$$

where the partition function for $(N-1)$ particles appears explicitly in the second term. After differentiating (A6) with respect to $x_{\vec{p}}$ and taking into account that this derivative of 
the partition function $Q_{N}^{n_{\vec{p}}=0}$ equals zero, we arrive at the following differential recurrence equations:

$$
x_{\vec{p}} \frac{\partial Q_{N}}{\partial x_{\vec{p}}}=x_{\vec{p}} Q_{N-1}+x_{\vec{p}}^{2} \frac{\partial Q_{N-1}}{\partial x_{\vec{p}}}
$$

The mean occupation numbers can be expressed through the derivative of the logarithm of the partition function $Q_{N}$ with respect to $x_{\vec{p}}$ (see Eq. (10)). So, Eq. (A7) for Bose particles reduces to the recurrence equations

$$
\left\langle n_{\vec{p}}\right\rangle_{N} Q_{N}=x_{\vec{p}} Q_{N-1}\left[1+\left\langle n_{\vec{p}}\right\rangle_{N-1}\right]
$$

where $Q_{0}=1$ and $\left\langle n_{\vec{p}}\right\rangle_{0}=0$ according to Eqs. (6) and (7), respectively.

Let us proceed now to the system of fermions. Plugging (A4) in (A2), we have for the partition functions of two systems with $N$ and $(N-1)$ particles

$$
\begin{gathered}
Q_{N}=Q_{N}^{n_{\vec{p}}=0}+x_{\vec{p}} Q_{N-1}^{n_{\vec{p}}=0} \\
Q_{N-1}=Q_{N-1}^{n_{\vec{p}}=0}+x_{\vec{p}} Q_{N-1-1}^{n_{\vec{p}}=0} .
\end{gathered}
$$

To get the differential equations for the system of fermions, let us differentiate Eqs. (A9) and (A10) with respect to $x_{\vec{p}}$, multiply the first equation by $x_{\vec{p}}$ and second one by $x_{\vec{p}}^{2}$ and sum these two :

$$
x_{\vec{p}} \frac{\partial Q_{N}}{\partial x_{\vec{p}}}=x_{\vec{p}} Q_{N-1}-x_{\vec{p}}^{2} \frac{\partial Q_{N-1}}{\partial x_{\vec{p}}} .
$$

Here we took into consideration that derivatives of $Q_{N}^{n_{\vec{p}}=0}$ and $Q_{N-1}^{n_{\vec{p}}=0}$ with respect to $x_{\vec{p}}$ equal zero. Combining (10) and (A11), we obtain the final recurrence equations for the Fermi system :

$$
\left\langle n_{\vec{p}}\right\rangle_{N} Q_{N}=x_{\vec{p}} Q_{N-1}\left[1-\left\langle n_{\vec{p}}\right\rangle_{N-1}\right] .
$$

The same initial condition as in the boson case are used here. One should note that Eqs. (A8) and (A12) for Bose and Fermi statistics differ only by the sign in front of the second term.

\section{APPENDIX B: MOMENTUM RESUMATION}

Using (74), let us present the sums in (45), (55), (59) and (63) in more convenient form

$$
\begin{aligned}
& f_{i l}=(\mp 1)^{l+1} e^{-\beta l E_{i}} \sum_{\vec{p}} e^{-\frac{\beta l \vec{p}^{2}}{2 m_{i}}} \\
& \overline{\varepsilon_{i l}}=(\mp 1)^{l+1} e^{-\beta l E_{i}} \sum_{\vec{p}} \frac{\vec{p}^{2}}{2 m_{i}} e^{-\frac{\beta l \vec{p}^{2}}{2 m_{i}}}+E_{i} f_{i l} \\
& \overline{\varepsilon_{i l}^{2}}=(\mp 1)^{l+1} e^{-\beta l E_{i}} \sum_{\vec{p}}\left(\frac{\vec{p}^{2}}{2 m_{i}}\right)^{2} e^{-\frac{\beta l \vec{p}^{2}}{2 m_{i}}}+2 E_{i} \overline{\varepsilon_{i l}}-E_{i}^{2} f_{i l} \\
& \overline{P_{i l}}=\frac{2}{3} \frac{\overline{\varepsilon_{i l}}}{V}-f_{i l}\left(\frac{\partial E_{i}}{\partial V}+\frac{2}{3} \frac{E_{i}}{V}\right) .
\end{aligned}
$$


The summation over momentum in the above Eqs. (B1)-(B4) can be written as

$$
\begin{aligned}
& \sum_{\vec{p}} e^{-\frac{\beta l \vec{p}^{2}}{2 m_{i}}}=\sum_{\vec{n}} e^{-\frac{\beta l(\Delta p)^{2}}{2 m_{i}} \vec{n}^{2}} \\
& \sum_{\vec{p}} \frac{\vec{p}^{2}}{2 m_{i}} e^{-\frac{\beta l \vec{p}^{2}}{2 m_{i}}}=\frac{(\Delta p)^{2}}{2 m_{i}} \sum_{\vec{n}} \vec{n}^{2} e^{-\frac{\beta l(\Delta p)^{2}}{2 m_{i}} \vec{n}^{2}} \\
& \sum_{\vec{p}}\left(\frac{\vec{p}^{2}}{2 m_{i}}\right)^{2} e^{-\frac{\beta l \vec{p}^{2}}{2 m_{i}}}=\left(\frac{(\Delta p)^{2}}{2 m_{i}}\right)^{2} \sum_{\vec{n}} \vec{n}^{4} e^{-\frac{\beta l(\Delta p)^{2}}{2 m_{i}} \vec{n}^{2}} .
\end{aligned}
$$

where $\vec{n} \equiv\left(n^{(x)}, n^{(y)}, v^{(z)}\right)$ and each $n^{(a)}$ runs over all integer numbers (see Eq. (11)). It is worthwhile to proceed to the momentum integration in $(\bar{B} 5)-(\bar{B} 7)$, though this is not valid in the whole region of temperature $T$ and volume $V$. The passage to the integration over momentum

$$
\sum_{\vec{p}} \ldots=\int \frac{d^{3} \vec{p}}{(\Delta p)^{3}} \ldots
$$

is possible under the condition

$$
\frac{(\Delta p)^{2} l}{2 m_{i} T} \leq 1
$$

In the opposite case, when

$$
\frac{(\Delta p)^{2} l}{2 m_{i} T}>1
$$

this passage can be erroneous and the sums $(\mathbb{B} 5)-(B 7)$ should be calculated numerically. If the condition $(\overline{\mathrm{B} 9})$ is satisfied, we have for integrals of $(\overline{\mathrm{B} 5})-(\overline{\mathrm{B} 7})$ :

$$
\begin{aligned}
\sum_{\vec{p}} e^{-\frac{\beta l \vec{p}^{2}}{2 m_{i}}} & =\frac{1}{l^{3 / 2}} \frac{V}{\lambda_{i}^{3}} \\
\sum_{\vec{p}} \frac{\vec{p}^{2}}{2 m_{i}} e^{-\frac{\beta l \vec{p}^{2}}{2 m_{i}}} & =\frac{3}{2} T \frac{1}{l^{5 / 2}} \frac{V}{\lambda_{i}^{3}} \\
\sum_{\vec{p}}\left(\frac{\vec{p}^{2}}{2 m_{i}}\right)^{2} e^{-\frac{\beta l \vec{p}^{2}}{2 m_{i}}} & =\frac{15}{4} T^{2} \frac{1}{l^{7 / 2}} \frac{V}{\lambda_{i}^{3}}
\end{aligned}
$$

where the thermodynamic wavelength for a fragment made from $i$ nucleons is

$$
\lambda_{i}=\left(\frac{2 \pi \hbar^{2}}{m_{i} T}\right)^{1 / 2}, \quad m_{i}=i m_{N}
$$

and $m_{N}$ stands here for the nucleon mass. After the substitution of (区11 $)-(\bar{B} 13)$ into $(\overline{B 1})-$ (B3), we obtain 


$$
\begin{aligned}
f_{i l} & =(\mp 1)^{l+1} e^{-\beta l E_{i}} \frac{1}{l^{3 / 2}} \frac{V}{\lambda_{i}^{3}} \\
\overline{\varepsilon_{i l}} & =\left(\frac{3}{2} T l^{-1}+E_{i}\right) f_{i l} \\
\overline{\varepsilon_{i l}^{2}} & =\left(\frac{15}{4} T^{2} l^{-2}+3 E_{i} T l^{-1}+E_{i}^{2}\right) f_{i l} .
\end{aligned}
$$

For calculating $(\mathbb{B} 5)-(\mathbb{B} 7)$ in the case $(\mathbb{B 1 0})$, let us present these sums in the form more suitable for computing. Let us introduce the function depending on the integer vector $\vec{n}$

$$
z_{i l}(\sigma)=\sum_{n=-\infty}^{\infty} n^{\sigma} e^{-\frac{\beta l(\Delta p)^{2}}{2 m_{i}} n^{2}}, \quad \sigma=0,2,4
$$

In this notation, the sums $(\mathbb{B 5})-(\bar{B} 7)$ are reduced to the following expressions :

$$
\begin{aligned}
\sum_{\vec{p}} e^{-\frac{\beta l \vec{p}^{2}}{2 m_{i}}} & =z_{i l}^{3}(0) \\
\sum_{\vec{p}} \frac{\vec{p}^{2}}{2 m_{i}} e^{-\frac{\beta l \vec{p}^{2}}{2 m_{i}}} & =3 z_{i l}(2) z_{i l}^{2}(0) \frac{(\Delta p)^{2}}{2 m_{i}} \\
\sum_{\vec{p}}\left(\frac{\vec{p}^{2}}{2 m_{i}}\right)^{2} e^{-\frac{\beta l \vec{p}^{2}}{2 m_{i}}} & =3 z_{i l}(0)\left[z_{i l}(4) z_{i l}(0)+2 z_{i l}^{2}(2)\right] \frac{(\Delta p)^{4}}{4 m_{i}^{2}}
\end{aligned}
$$

To calculate them, it is sufficient to know functions $z_{i l}(0), z_{i l}(2)$ and $z_{i l}(4)$ in the limited range of values $n$, because exponents go fast to zero with increasing the absolute value of $n$. 


\section{REFERENCES}

[1] W.A. Friedman, Phys. Rev. C 42 (1990) 667.

[2] X. Campi, J. Phys. A 19 (1986) L917; Phys. Lett. B208 (1988) 351.

[3] W. Bauer, Phys. Rev. C 38 (1986) 1297;

W. Bauer et al, Nucl. Phys. A 452 (1986) 699;

W. Bauer and A. Botvina, Phys. Rev. C 52 (1995) 1750.

[4] J. Randrup and S. Koonin, Nucl. Phys. A 356 (1981) 321.

[5] D.H.E. Gross, Rep. Prog. Phys. 53 (1990) 605.

[6] J.P. Bondorf, A.S. Botvina, A.S. Iljinov, I.N. Mishustin and K. Sneppen, Phys. Rep. 257 (1995) 133.

[7] A. Le Fevre, M. Płoszajczak and V.D. Toneev, Phys. Rev. C 60 (1999) R051602.

[8] J. Aichelin, Phys. Rep. 202 (1991) 233.

[9] R. Botet and M. Płoszajczak, Int. J. Mod. Phys. E3 (1994) 1033.

[10] H. Feldmeier and J. Schnack, Prog. Particle Nucl. Phys. 39 (1997) 393.

[11] A. Ohnishi and J. Randrup, Phys. Lett. B394 (1997) 260.

[12] D. Kiderlen and P. Danielewicz, Nucl. Phys. A 620 (1997) 346.

[13] P.R. Subramanian et al., J. Phys. G 7 (1982) L241.

[14] D. Hahn and H. Stoecker, Nucl. Phys. A 476 (1988) 719.

[15] R.K. Tripathy and L.W. Townsend, Phys. Rev. C 50 (1994) R7.

[16] J. Konopka, H. Graf, H. Stoecker and W. Greiner, Phys. Rev. C 50 (1994) 2085.

[17] F. Gulminelli and D. Durand, Nucl. Phys. A 615 (1997) 117.

[18] K.C. Chase and A.Z. Mekjian, Phys. Rev. C 49 (1994) 2164.

[19] K.C. Chase and A.Z. Mekjian, Phys. Rev. C 52 (1995) R2339.

[20] J. Riordan, An Introduction to Combinatorial Analysis (Wiley, New York, 1958).

[21] R. Hanbury-Brown and R.Q. Twiss, Nature (London) 177 (1957) 27;

G. Goldhaber, S. Goldhaber, W. Lee and A. Pais, Phys. Rev. 120 (1960) 300.

[22] P. Bożek, M. Płoszajczak and R. Botet, Phys. Rep. 252 (1995) 101.

[23] J. Zimanyi and T. Csórgo, Heavy Ion Phys. 9 (1999) 241.

[24] Ch.K. Chow and Th.D. Cohen, quant-ph/9911101.

[25] K. Huang, Statistical Mechanics (John Wiley \& Sons Inc., 1963).

[26] M. Abramowitz and I. Stegun, Handbook of Mathematics Functions, Nat. Bur. Stand. Appl. Math. Ser. No. 55, (U.S. GPO, Washington, D.C., 1965).

[27] S. Pratt, nucl-th/9905055.

[28] L.D. Landau and E.M. Lifshitz, Statistical Physics (Pergamon Press, 1985).

[29] J.P. Bondorf, R. Donangelo, I.N. Mishustin, C.J. Pethick, H. Schulz and K. Sneppen, Nucl. Phys. A 443 (1985) 321.

[30] J.P. Bondorf, R. Donangelo, I.N. Mishustin, and H. Schulz, Nucl. Phys. A 444 (1985) 460.

[31] A.Z. Mekjian, Phys. Rev. Lett. 64 (1990) 2125; Phys. Rev. C 41 (1990) 2103.

[32] S.J. Li and A.Z. Mekjian, Phys. Lett. A 149 (1990) 7; Phys. Rev. C 45 (1992) 365; Phys. Rev. C 45 (1992) 1284; Phys. Rev. C 47 (1993) 2266; Phys. Rev. C 50 (1994) 3025; Phys. Rev. C 56 (1997) 2621.

[33] P. Marmier and E. Sheldon, Physics of Nuclei and Particles (Academic, New York, 1970), vol.II, p.886.

[34] D.H Rischke, M.I. Gorenstein, H. Stoecker and W. Greiner, Z. Phys. C 51 (1991) 485. 
[35] Saeeduddin, Phys. Lett. B 341 (1995) 361.

[36] A.A. Shanenko,E.P. Yukalova and V.I. Yukalov, Int. J. Mod. Phys. B 10 (1996) 669.

[37] A. Majumder and S. Das Gupta, Phys. Rev. C 59 (1999) 845.

[38] A.H. Raduta and A.R. Raduta, nucl-th/9908089.

[39] A.J. Cole, D. Heuer, and M. Charvet, Phys. Rev. C 55 (1997) 2978.

[40] M.I. Gorenstein, A.P. Kostyk and Ya.D. Krivenko, J. Phys. G: Nucl. Part. Phys. 25 (1999) L75.

[41] C.A Ogilvie at al., Phys. Rev. Lett. 67 (1991) 1214;

J. Hubele et al., Z. Physik A340 (1991) 263; Phys. Rev. C 46 (1992) R1577.

[42] J. Pochodzalla at al., Phys. Rev. Lett. 75 (1995) 1040.

[43] S. Pratt and S.D. Gupta, nucl-th/9903006.

[44] S.D. Gupta, A. Majumder, S. Pratt and A. Mekjian, nucl-th/9903007.

[45] H. Müller and B. Serot, Phys. Rev. C 52 (1995) 2972.

[46] A. Hauger et al., Phys. Rev. Lett. 77 (1996) 235.

[47] K.C. Chase and A.Z. Mekjian, Phys. Rev. C 50 (1994) 2078.

[48] A.S. Parvan, K.K. Gudima and V.D. Toneev, Yad. Fiz. 62 (1999) 1593. 


\section{Figure captures}

Fig. 1. The mean occupation numbers $\left\langle\nu_{\vec{p} i}\right\rangle$ of fragments of mass $i$ for $A=200$ system at temperatures of 10 and $15 \mathrm{MeV}$. The dashed, dotted and continuous curves correspond to the QSM result obtained under the assumption that all fragments are bosons, fermions or Boltzmann particles, respectively. The system volume is $V / V_{0}=3$ and $\Delta p=90 \mathrm{MeV} / \mathrm{c}$.

Fig. 2. The temperature dependence of the mean multiplicity $\left\langle N_{i}\right\rangle$ for fragment of mass $i$ in the fragmenting system of size $A=200$. For further details, see the caption of Fig. 1 . The results are obtained for the two values of the system volume.

Fig. 3.The temperature dependence of the total mean multiplicity $\langle m\rangle$ and IMF multiplicity $\langle M\rangle$ for systems with $A=100$ and 200. For further details see the caption of Fig. 2.

Fig. 4. The temperature dependence of the occupation number correlation $\eta_{i}$ of the fragment species $i$ for the system with $A=200$ at two different values of parameter $V / V_{0}$. For further details see the caption of Fig. 2.

Fig. 5. The temperature dependence of the occupation-number correlation parameter summed over all fragments $\eta(m)$ and IMF's $\eta(M)$ for $A=200$. For further details see the caption of Fig. 4.

Fig. 6. The temperature dependence of the shifted multiplicity dispersion $\gamma_{i}$ of the fragment species $i$ for the system with $A=200$ at two values of the freeze-out volume parameter $V / V_{0}$. For further details see the caption of Fig. 2.

Fig. 7. The temperature dependence of the shifted multiplicity dispersion summed over all fragments $\gamma(m)$ and IMF's $\gamma(M)$ for the system with $A=200$. For further details see the caption of Fig. 4.

Fig. 8. The heat capacity and the total energy vs. temperature for systems with $A=200$ (solid line) and 100 (dashed) nucleons at two different values of the volume parameter $V / V_{0}$. Dotted curves are calculated for $A=200$ system neglecting the Coulomb interaction energy. The results for Bose, Fermi and Boltzmann statistics coincide with each other with the accuracy comparable to the line thickness.

Fig. 9. The pressure as a function of the volume at the constant temperature $T$ for the systems size $A=100$ and 200 with (l.h.s.) and without (r.h.s.) taking into account the Coulomb interaction energy. The results for Bose, Fermi and Boltzmann statistics coincide with each other with the accuracy comparable to the line thickness.

Fig. 10. The IMF multiplicity distributions for systems with $A=200$ and 100 nucleons at the given temperature $T$ for two different values of the volume parameter : $V / V_{0}=6$ (lower panels) and $V / V_{0}=3$ (upper panels). Open squares, open circles and filled triangles are the QSM result obtained under the assumption that all fragments are bosons, fermions 
or Boltzmann particles, respectively.

Fig. 11. The total fragment multiplicity distributions. For further details see the caption of Fig. 10. 

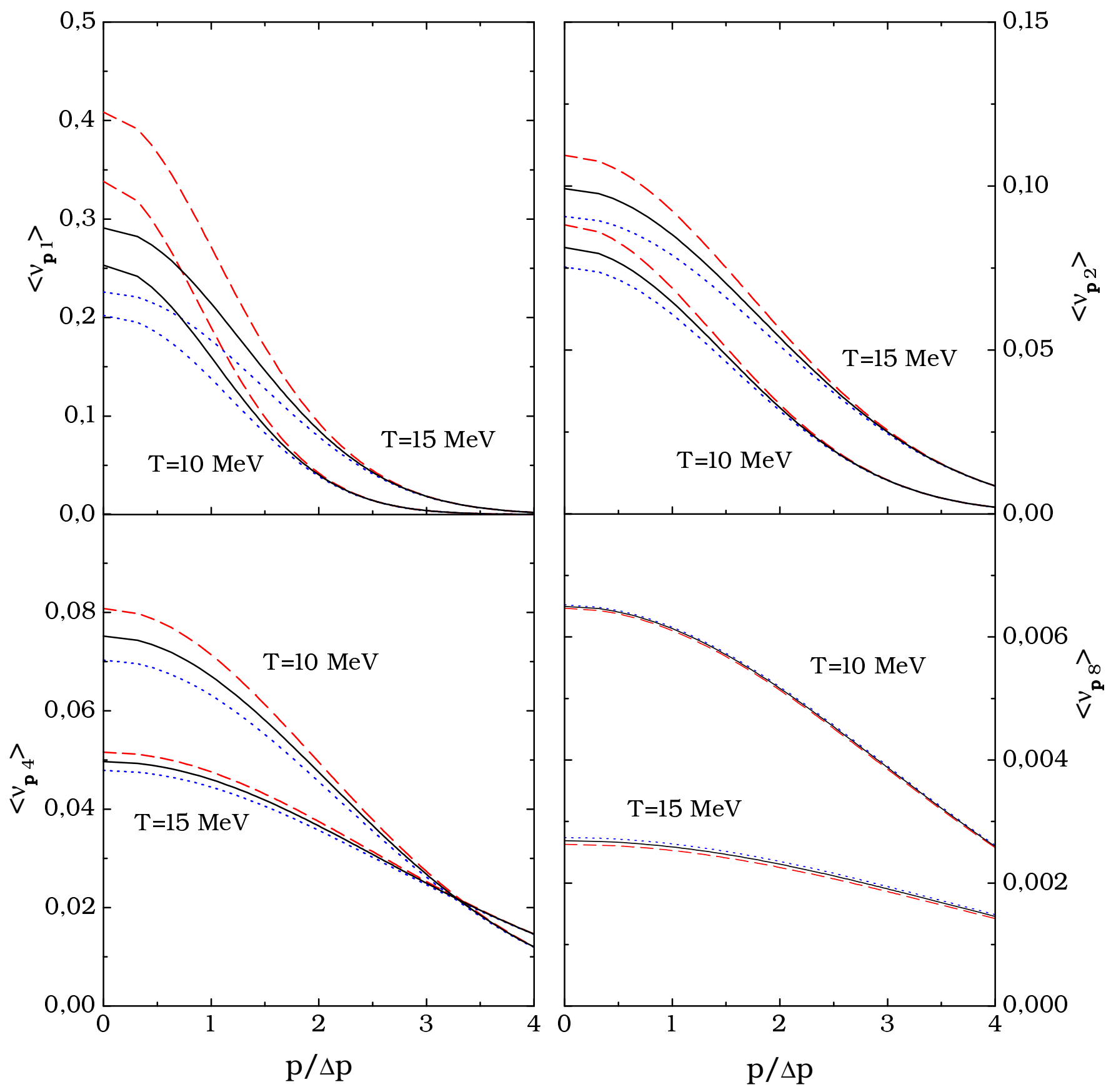

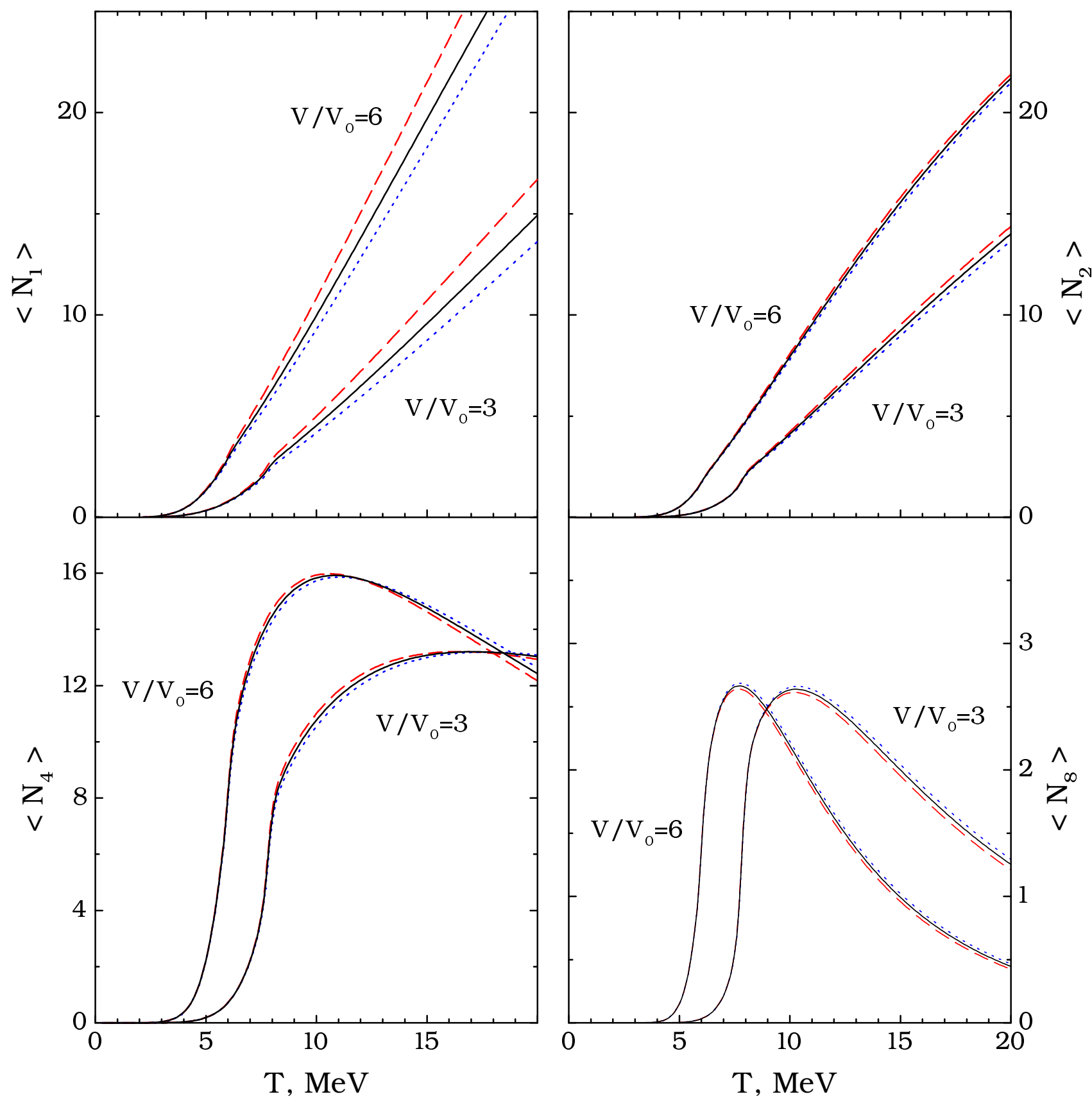

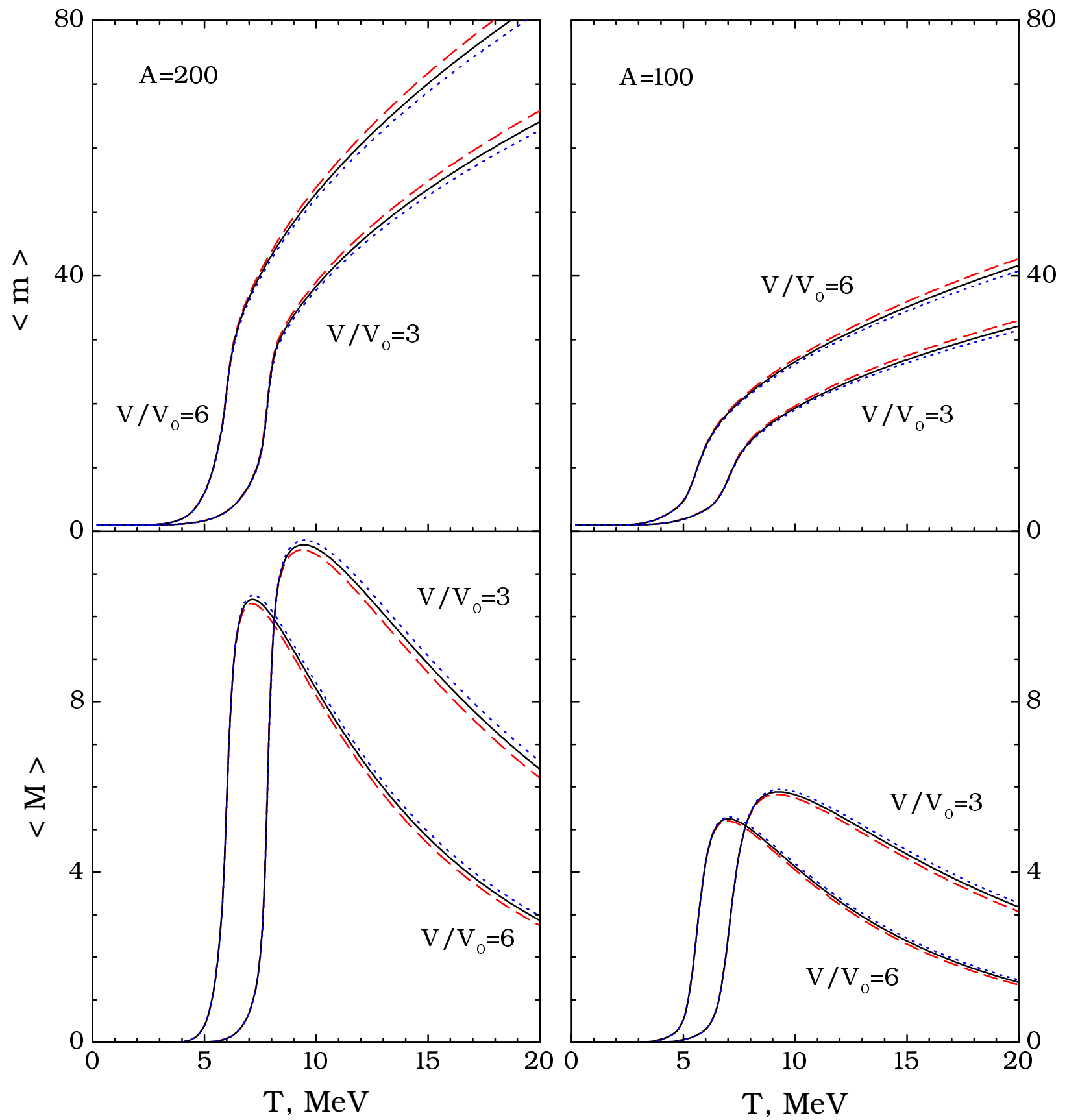

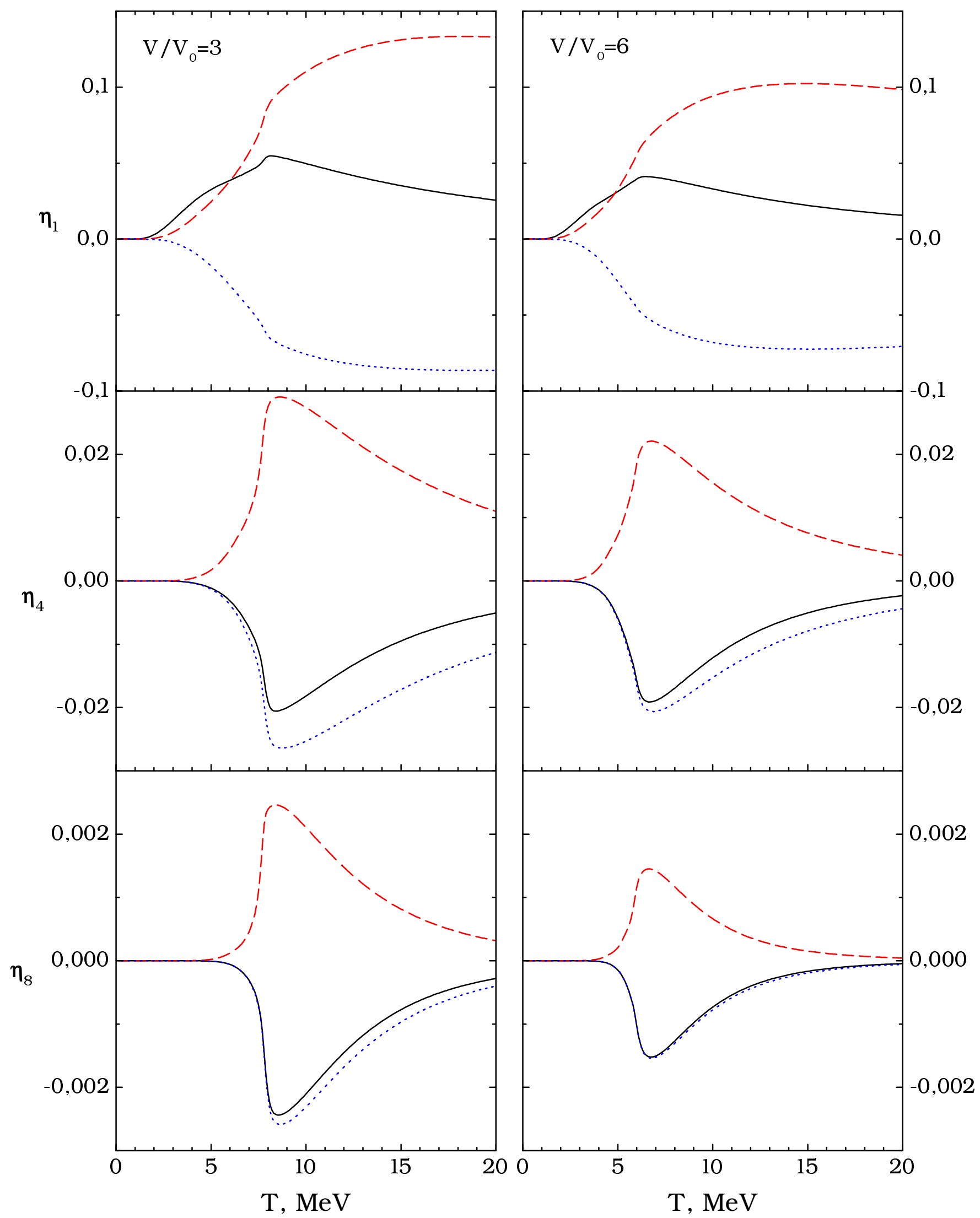


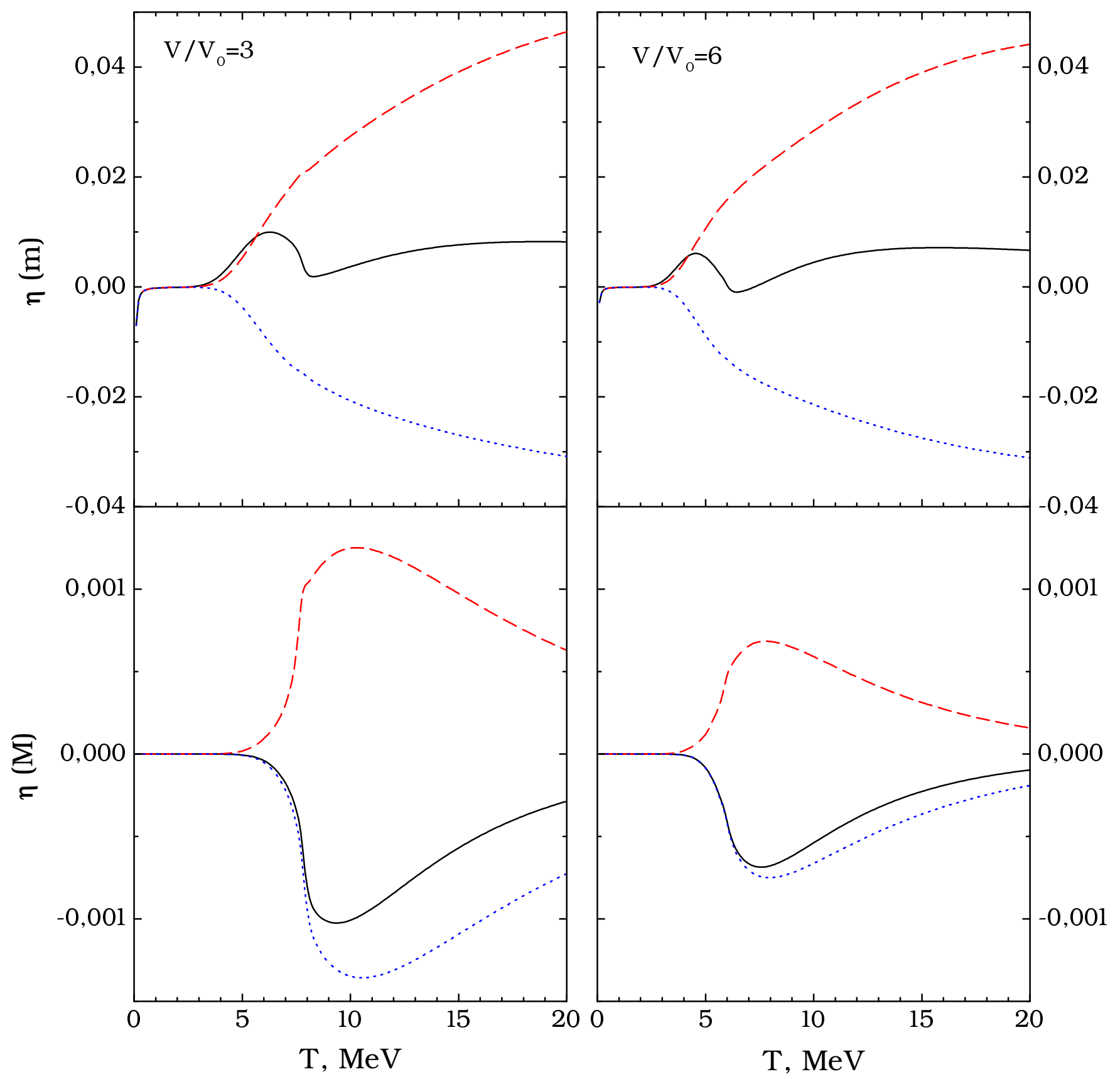



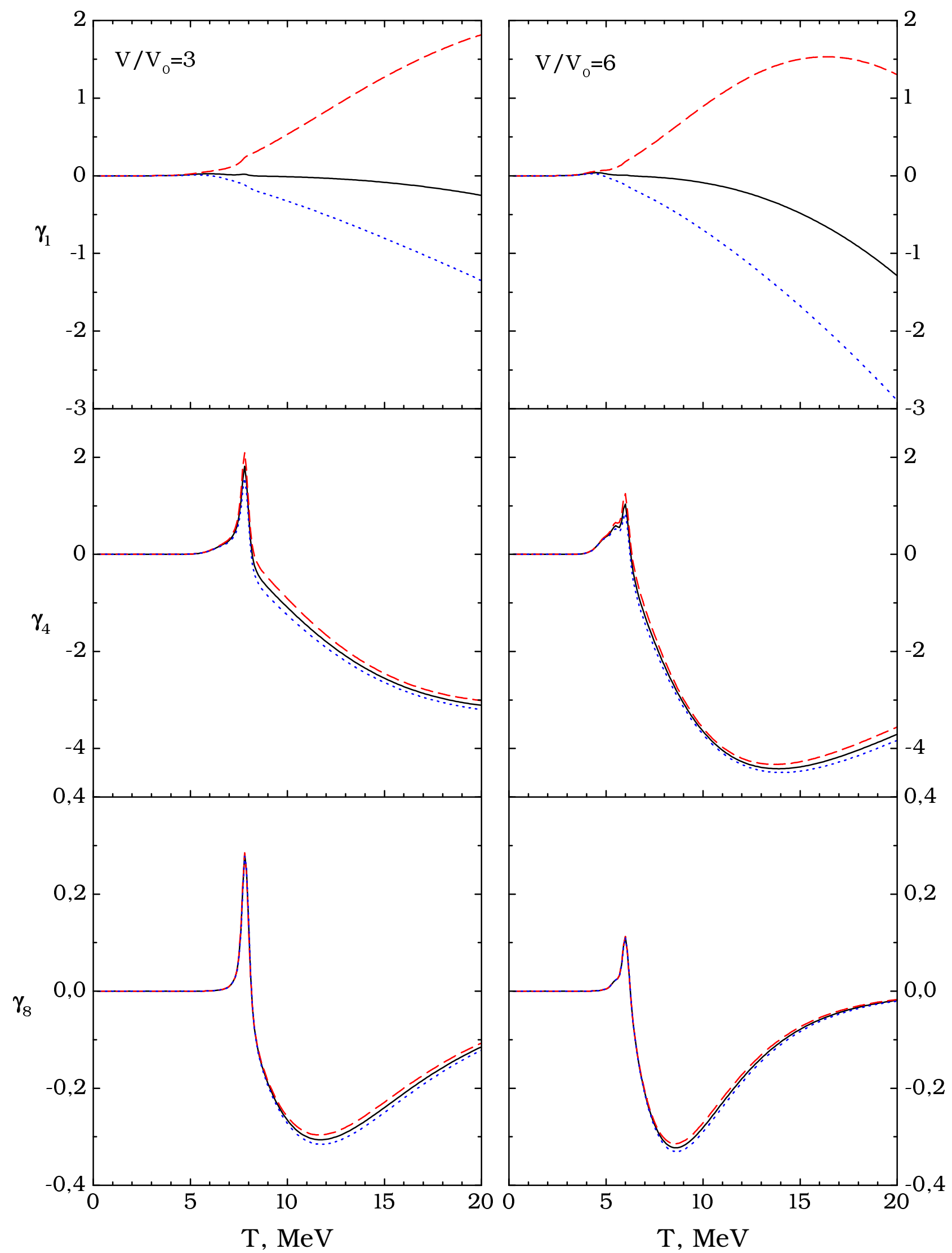

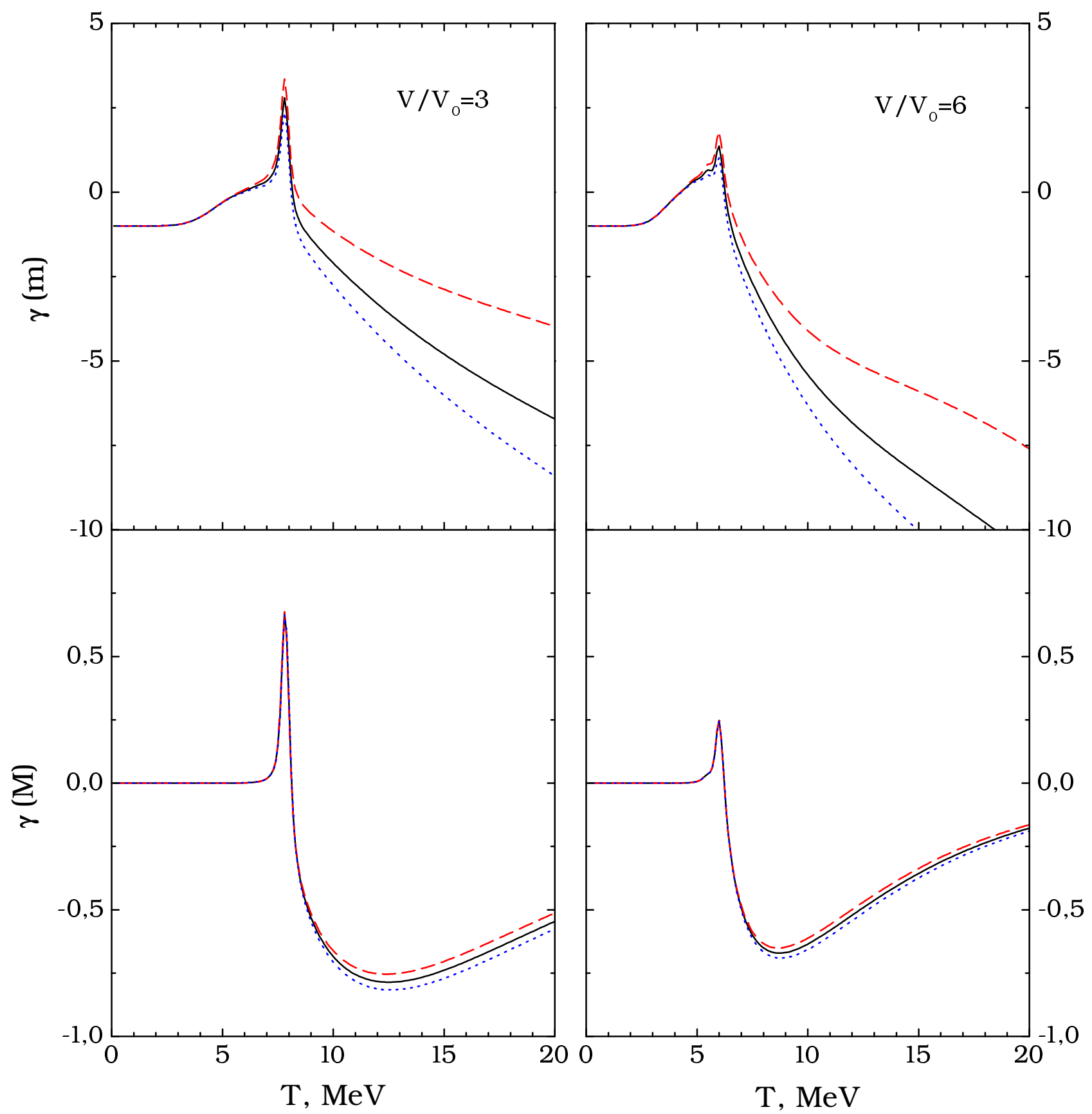

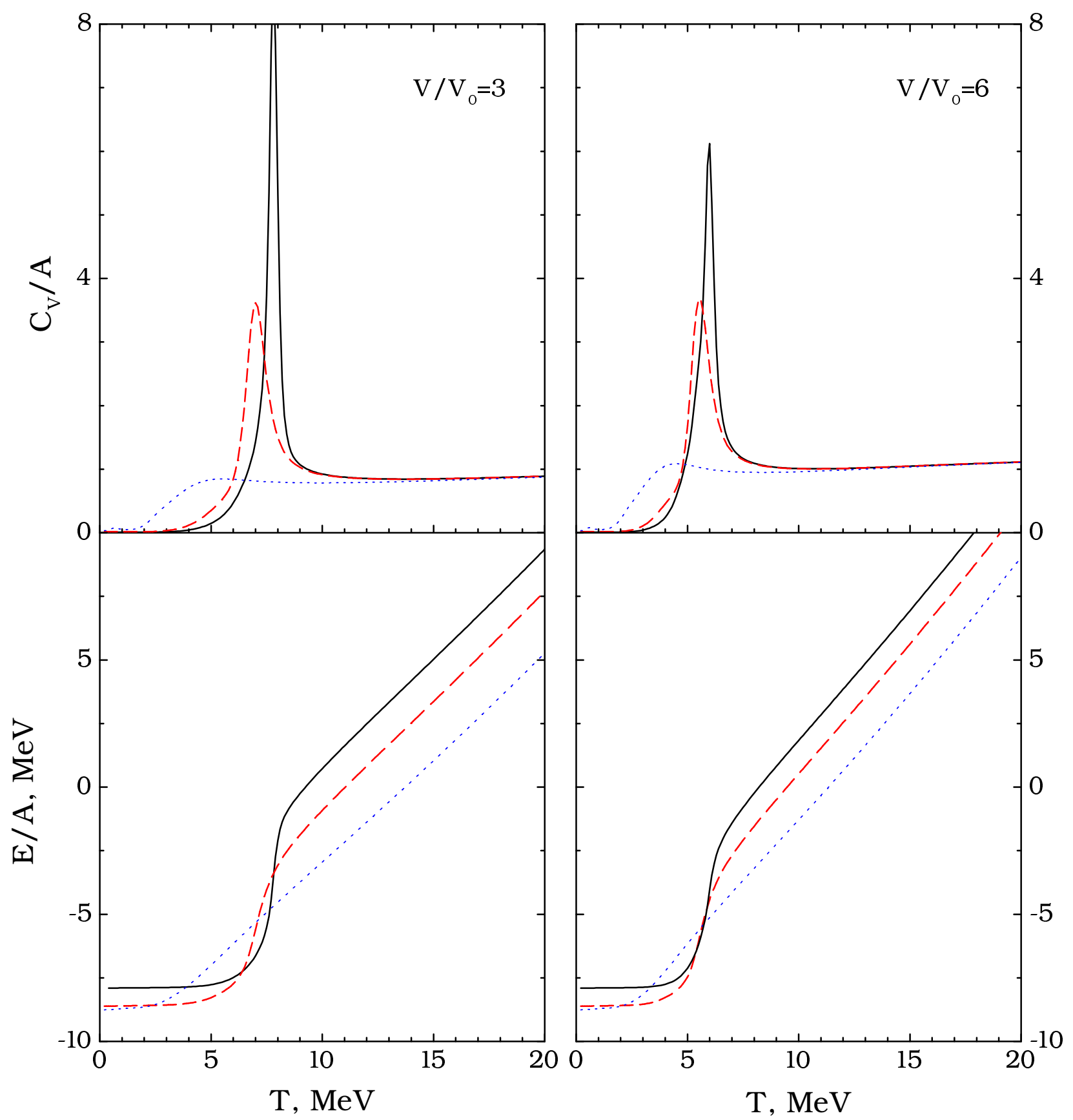

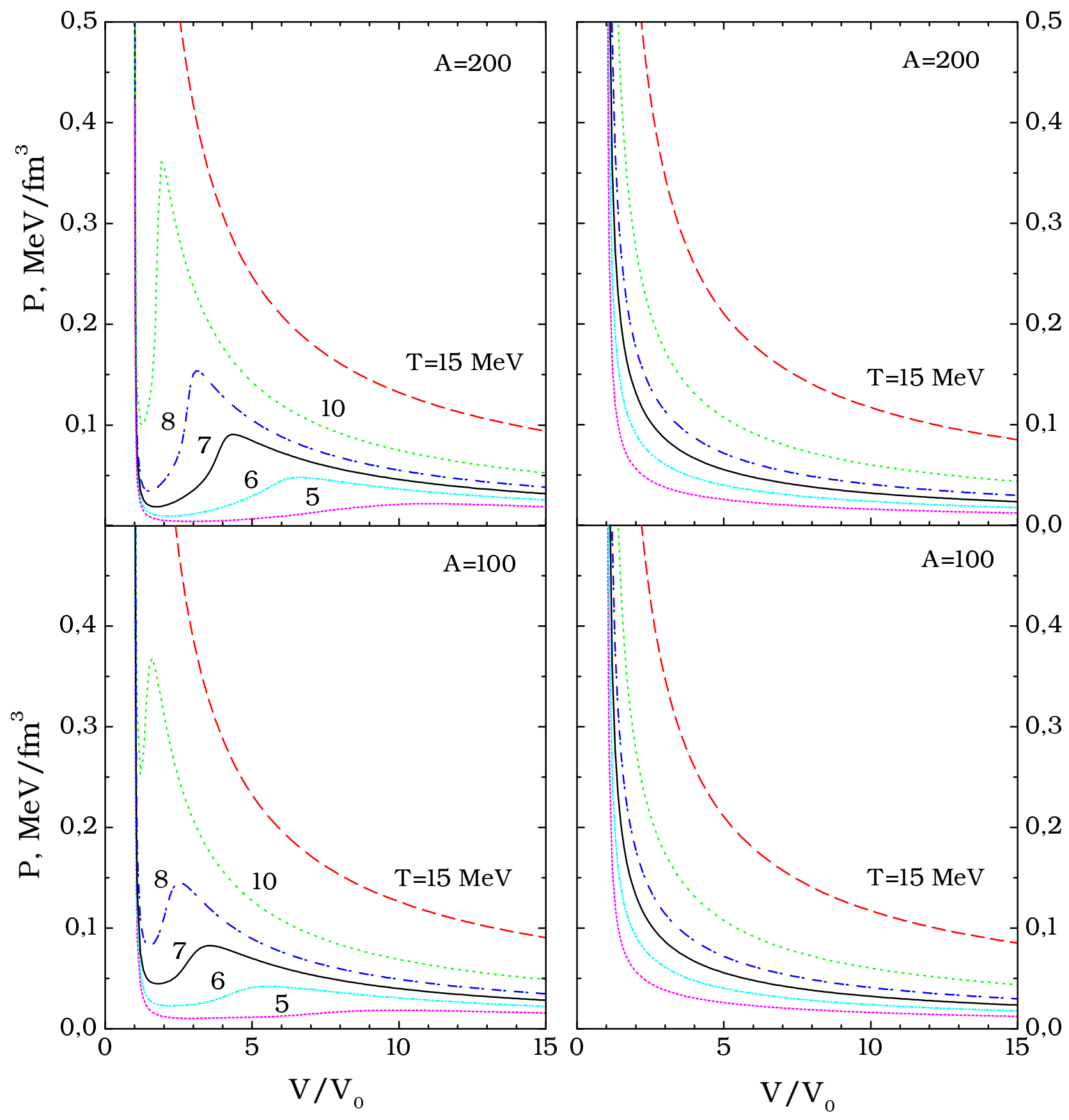


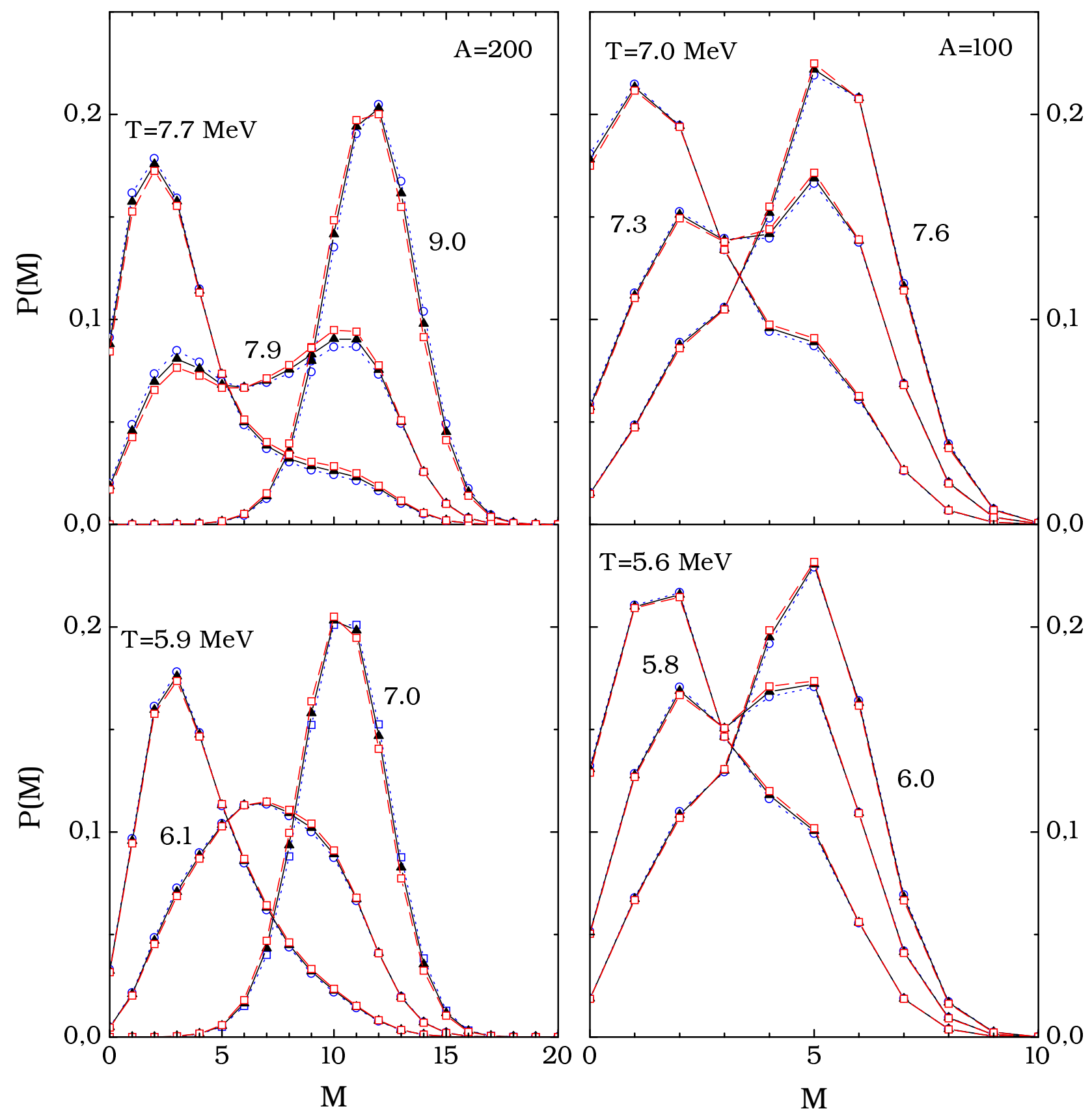




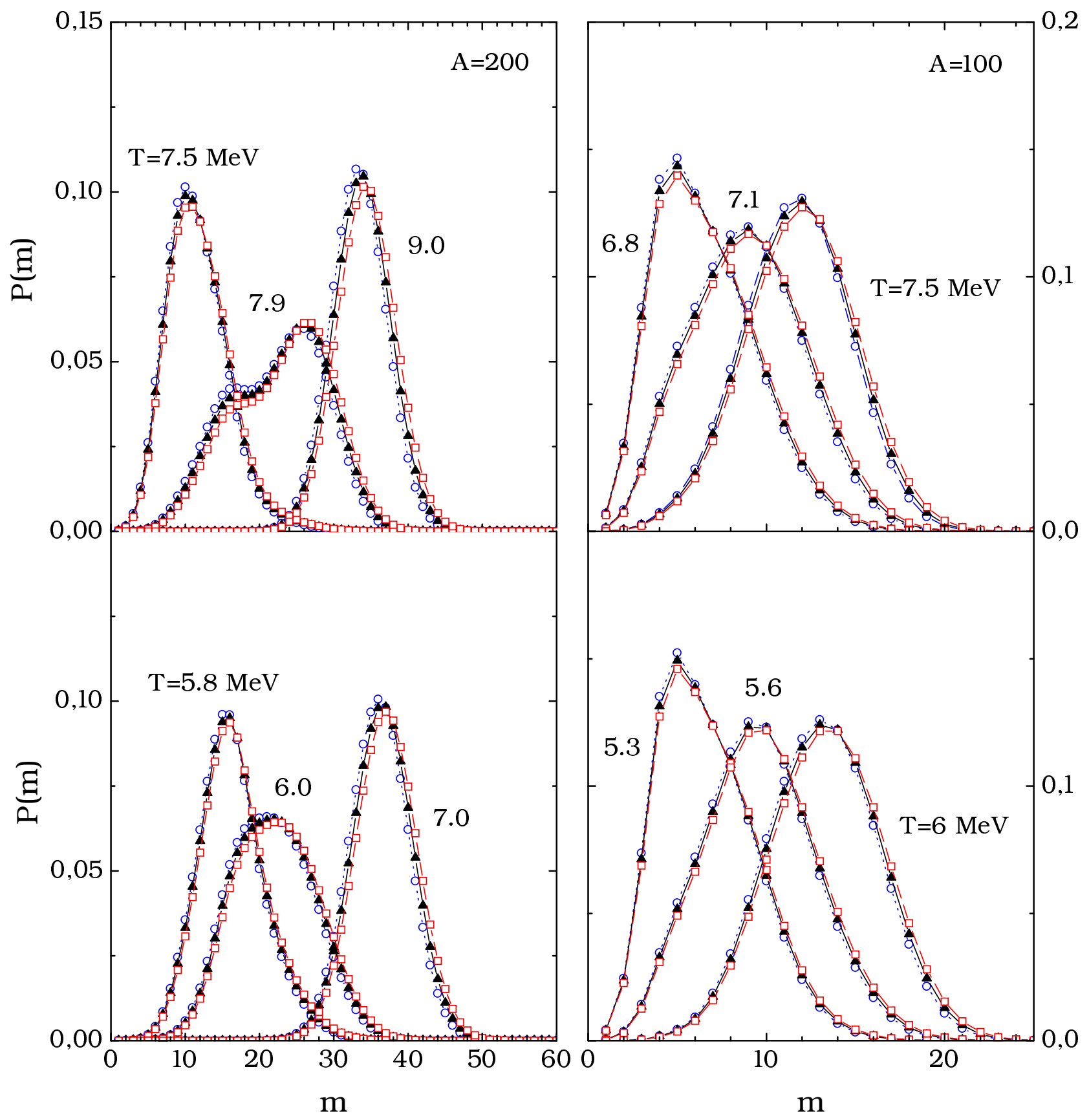

